\title{
Summary of the NASA Science Instrument, Observatory and Sensor System (SIOSS) Technology Assessment
}

H. Philip Stahl, NASA Marshall Space Flight Center

Rich Barney, NASA Goddard Space Flight Center

Jill Bauman, NASA Ames Research Center

Lee Feinberg, NASA Goddard Space Flight Center

Dan Mccleese, Jet Propulsion Laboratory

Upendra Singh, NASA Langley Research Center 


\section{AGENDA}

Office of Chief Technologist (OCT) Technology Area Roadmap

Science Instrument, Observatory and Sensor Systems TA

Needs Assessment

Technology Area Breakdown Structure (TABS)

Technology Development Roadmaps

Top Challenges

Interdependencies with other TAs and Government Agencies

Budget Recommendations

Conclusions 
NASA Office of Chief Technologist

Aero-Space Technology Area Roadmap

(A-STAR) 


\section{Aero-Space Technology Area Roadmap (A-STAR)}

July 2010, NASA Office of Chief Technologist (OCT) initiated an activity to create and maintain a NASA integrated roadmap for 15 key technology areas which recommend an overall technology investment strategy and prioritize NASA's technology programs to meet NASA's strategic goals.

Initial reports were presented to the National Research Council who are currently collecting public input and preparing reviews of each Roadmap.

Roadmaps will be updated annually and externally reviewed every 4 years consistent with the Agency's Strategic Plans. 


\section{A-STAR Process}

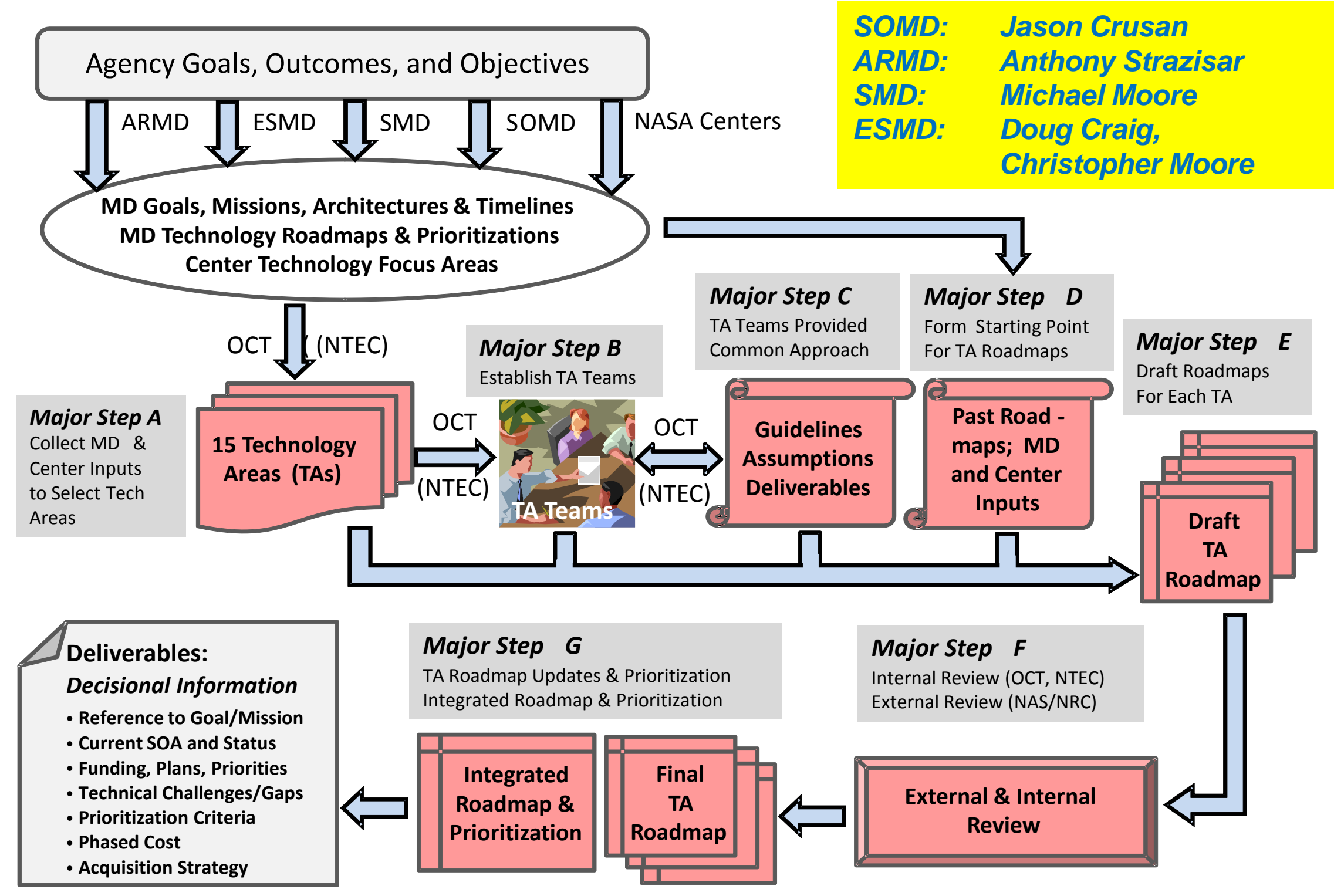




\section{Technology Assessment Areas}

TA1: Launch Propulsion Systems

TA2: In-Space Propulsion Systems

TA3: $\quad$ Space Power and Energy Storage Systems

TA4: Robotics, Tele-robotics, and Autonomous Systems

TA5: Communication and Navigation Systems

TA6: Human Health, Life Support and Habitation Systems

TA7: Human Exploration Destination Systems

TA8: Scientific Instruments, Observatories, and Sensor Systems

TA9: Entry, Descent, and Landing Systems

TA10: Nanotechnology

TA11: Modeling, Simulation, Information Technology, and Processing

TA12: Materials, Structural \& Mechanical Systems, and Manufacturing

TA13: Ground and Launch Systems Processing

TA14: Thermal Management Systems

TA15: Aeronautics 


\section{Goals and Benefits}

Develop clear NASA technology portfolio recommendations

Prioritize current needs

Define development plans

Identify alternative paths

Reveal interrelationships of between various technologies

Transparency in government technology investments

Ensure needs of all NASA Mission Directorates are included

Credibility for planned NASA technology programs

Coordinate with other Government agencies

Broad-based input from non-government parties 


\section{Charge to TA Teams}

Review, document, and organize the existing roadmaps and technology portfolios.

Collect input from key Center subject matter experts, program offices and Mission Directorates.

Take into account:

US aeronautics and space policy;

NASA Mission Directorate strategic goals and plans;

Existing Design Reference Missions, architectures and timelines; and Past NASA technology and capability roadmaps.

Recommend 10-yr Budget to Mature Technology to TRL6 


\section{Technology Assessment Content}

Define a breakdown structure that organizes and identifies the TA Identify and organize all systems/technologies involved in the TA using a 20-year horizon

Describe the state-of-the-art (SOA) for each system

Identify the various paths to achieve performance goals

Identify NASA planned level of investment Assess gaps and overlaps across planned activities

Identify alternate technology pathways

Identify key challenges required to achieve goals 


\section{Technology Assessment \#8:}

Science Instruments, Observatories and Sensor Systems

(SIOSS) 


\section{TA8 Roadmap Team}

Rich Barney (GSFC), Division Chief, Instrument Systems and Technology Division. Co-chaired 2005 NASA Science Instruments and Sensors Capability Roadmap.

Phil Stahl (MSFC), Senior Optical Physicists

Optical Components Technical Lead for James Webb Space Telescope;

Mirror Technology Days in the Government;

Advanced Optical Systems SBIR Subtopic Manager;

2005 Advanced Observatories and Telescopes Capability Roadmap.

Upendra Singh (LaRC), Chief Technologist, Engineering Directorate.

Principal Investigator for NASA Laser Risk Reduction Program (2002-2010)

Dan Mccleese (JPL), Chief Scientist

Principal Investigator of Mars Climate Sounder instrument on Mars Reconnaissance Orbiter.

Jill Bauman (ARC), Associate Director of Science for Mission Concepts.

Lee Feinberg (GSFC), Chief Large Optics System Engineer

JWST OTE Manager.

Co-chaired 2005Advanced Telescopes and Observatories Capability Roadmap. 


\section{SIOSS}

SIOSS roadmap addresses technology needs to achieve NASA's highest priority objectives - not only for the Science Mission Directorate (SMD), but for all of NASA.

SIOSS Team employed a multi-step process.

- Performed an SMD needs assessment;

- Consolidated the identified technology needs into broad categories and organized them into a Technology Area Breakdown Structure (TABS);

- Generated technology development roadmaps for each TABS element;

- Investigated interdependencies with other TA Areas as well as the needs of Other Government Agencies. 


\section{SMD Needs Assessment}

First step was to review governing documents (such as Decadal Surveys, roadmaps, and science plans) for each Science Mission Directorate (SMD) divisions: Astrophysics, Earth Science, Heliophysics, and Planetary Science:

2010 Science Plan, NASA Science Mission Directorate, 2010

Agency Mission Planning Manifest, 2010

New Worlds, New Horizons in Astronomy and Astrophysics, NRC Decadal Survey, 2010

Panel Reports: - New Worlds, New Horizons in Astronomy and Astrophysics, NRC Decadal

Survey, 2010

Heliophysics, The Solar and Space Physics of a New ERA, Heliophysics Roadmap Team

Report to the NASA Advisory Council, 2009

Earth Science and Applications from Space, NRC Decadal Survey, 2007

New Frontiers in the Solar Systems, NRC Planetary Decadal Survey, 2003

The Sun to the Earth - and Beyond, NRC Heliophysics Decadal Survey, 2003

Advanced Telescopes and Observatories, APIO, 2005

Science Instruments and Sensors Capability, APIO, 2005 


\section{Astrophysics Technology Needs}

National Academy 2010 Decadal Report recommended missions and technology-development programs, (with need date):

Wide Field Infrared Survey Telescope (WFIRST), 2018

Explorer Program, 2019/2023

Laser Interferometer Space Antenna (LISA), 2024

International X-ray Observatory (IXO), mid/late 2020s

New Worlds Technology Development Program, mid/late 2020s

Epoch of Inflation Technology Development Program, mid/late 2020s

U.S. Contribution to the JAXA-ESA SPICA Mission, 2017

UV-Optical Space Capability Technology Development Program, mid/late 2020s

TRL3-to-5 Intermediate Technology Development Program

All can be enhanced or enabled by technology development to reduce cost, schedule, and performance risks. 


\section{SMD Needs Assessment}

Detailed listings of technology needs for each SMD division were tabulated which enable either:

planned SMD missions ('pull technology') or

emerging measurement techniques necessary for new scientific discovery

('push technology').

These lists were then reviewed and refined by individual mission and technology-development stakeholders. 
Table 2.2.1.1 - 1 Summary of Astrophysics Technology Needs

\begin{tabular}{|c|c|c|c|c|c|c|}
\hline Mission & Technology & Metric & State of Art & Need & Start & TRL6 \\
\hline WFIRST & NIR detectors & $\begin{array}{l}\text { Pixel array } \\
\text { Pixel size }\end{array}$ & $\begin{array}{l}2 \mathrm{k} \times 2 \mathrm{k} \\
18 \mu \mathrm{m}\end{array}$ & $\begin{array}{l}4 \mathrm{k} \mathrm{x} 4 \mathrm{k} \\
10 \mu \mathrm{m}\end{array}$ & 2012 & 2014 \\
\hline $\begin{array}{l}\text { UVOTP } \\
\text { Push }\end{array}$ & $\begin{array}{l}\text { Detector arrays: } \\
\text { Low noise }\end{array}$ & $\begin{array}{l}\text { Pixel } \\
\text { QE UV } \\
\text { QE Visible } \\
\text { Rad Hard } \\
\end{array}$ & $2 \mathrm{k} \times 2 \mathrm{k}$ & $\begin{array}{l}4 \mathrm{k} \mathrm{x} 4 \mathrm{k} \\
>0.590-300 \mathrm{~nm} \\
>0.8300-900 \mathrm{~nm} \\
50 \text { to } 200 \mathrm{kRad}\end{array}$ & 2012 & 2020 \\
\hline $\begin{array}{l}\text { NWTP } \\
\text { Push }\end{array}$ & Photon counting arrays & $\begin{array}{l}\text { Pixel array visible } \\
\text { Visible QE } \\
\text { Pixel array NIR }\end{array}$ & $\begin{array}{l}512 \times 512 \\
80 \% 450-750 \mathrm{~nm} \\
128 \times 128\end{array}$ & $\begin{array}{l}1 \mathrm{k} \times 1 \mathrm{k} \\
>80 \% 450-900 \mathrm{~nm} \\
256 \times 256\end{array}$ & 2011 & 2020 \\
\hline $\begin{array}{l}\text { SPICA } \\
\text { ITP } \\
\text { Push }\end{array}$ & Far-IR detector arrays & $\begin{array}{l}\text { Sens. (NEP W/ } / \sqrt{H z} \text { ) } \\
\text { Wavelength } \\
\text { Pixels }\end{array}$ & $\begin{array}{l}1 \mathrm{e}-18 \\
>250 \mu \mathrm{m} \\
256\end{array}$ & $\begin{array}{l}3 \mathrm{e}-20 \\
35-430 \mu \mathrm{m} \\
1 \mathrm{k} \times 1 \mathrm{k}\end{array}$ & 2011 & $\begin{array}{l}2015 \\
2020\end{array}$ \\
\hline $\begin{array}{l}\text { IXO } \\
\text { Push }\end{array}$ & X-ray detectors & $\begin{array}{l}\text { Pixel array } \\
\text { Noise } \\
\text { QE } \\
\text { Frame rate }\end{array}$ & $\begin{array}{l}10-15 \mathrm{e}^{-} \mathrm{RMS} \\
100 \mathrm{kHz} @ 2 \mathrm{e}^{-}\end{array}$ & $\begin{array}{l}40 \text { x } 40 \text { TES } \\
2-4 \mathrm{e}^{-} \mathrm{RMS} \\
>0.70 .3-8 \mathrm{keV} \\
0.5-1 \mathrm{MHz} @ 2 \mathrm{e}^{-}\end{array}$ & 2011 & 2015 \\
\hline $\begin{array}{l}\text { WFIRST } \\
\text { IXO }\end{array}$ & Detector ASIC & $\begin{array}{l}\text { Speed @ low noise } \\
\text { Rad tolerance }\end{array}$ & $\begin{array}{l}100 \mathrm{kHz} \\
14 \mathrm{krad}\end{array}$ & $\begin{array}{l}0.5-1 \mathrm{MHz} \\
55 \mathrm{krad}\end{array}$ & 2011 & 2013 \\
\hline NWTP & $\begin{array}{l}\text { Visible Starlight } \\
\text { suppression: } \\
\text { coronagraph or } \\
\text { occulter }\end{array}$ & $\begin{array}{l}\text { Contrast } \\
\text { Contrast stability } \\
\text { Passband } \\
\text { Inner Working Angle }\end{array}$ & $\begin{array}{l}>1 \times 10^{-9} \\
--- \\
10 \%, 760-840 \mathrm{~nm} \\
4 \lambda / \mathrm{D}\end{array}$ & $\begin{array}{l}<1 \times 10^{-10} \\
1 \times 10^{-11} / \text { image } \\
20 \%, \text { at } V, I, \text { and } R \\
2 \lambda / \mathrm{D}-3 \lambda / \mathrm{D}\end{array}$ & $\begin{array}{l}2011 \\
2011\end{array}$ & $\begin{array}{l}2016 \\
2020\end{array}$ \\
\hline NWTP & $\begin{array}{l}\text { Mid-IR Starlight } \\
\text { suppres: interferometer }\end{array}$ & $\begin{array}{l}\text { Contrast } \\
\text { Passband mid-IR }\end{array}$ & $\begin{array}{l}1.65 \times 10^{-5}, \text { laser } \\
30 \% \text { at } 10 \mu \mathrm{m}\end{array}$ & $\begin{array}{l}<1 \times 10^{-7} \text {, broadband } \\
>50 \% 8 \mu \mathrm{m}\end{array}$ & $\begin{array}{l}2011 \\
2011\end{array}$ & $\begin{array}{l}2016 \\
2020\end{array}$ \\
\hline $\begin{array}{l}\text { NWTP } \\
\text { UVOTP }\end{array}$ & $\begin{array}{l}\text { Active WFSC; } \\
\text { Deformable Mirrors }\end{array}$ & $\begin{array}{l}\text { Sensing } \\
\text { Control (Actuators) }\end{array}$ & $\begin{array}{l}\lambda / 10,000 \mathrm{rms} \\
32 \times 32\end{array}$ & $\begin{array}{l}<\lambda / 10,000 \mathrm{rms} \\
128 \times 128\end{array}$ & 2011 & 2020 \\
\hline IXO & XGS CAT grating & Facet size; Throughput & $3 \times 3 \mathrm{~mm} ; 5 \%$ & $60 \times 60 \mathrm{~mm} ; 45 \%$ & 2010 & 2014 \\
\hline Various & Filters \& coatings & Reflect/transmit; temp & & & 2011 & 2020 \\
\hline Various & Spectroscopy & Spectral range/resolve & & & 2011 & 2020 \\
\hline $\begin{array}{l}\text { SPICA } \\
\text { IXO }\end{array}$ & $\begin{array}{l}\text { Continuous sub-K } \\
\text { refrigerator }\end{array}$ & $\begin{array}{l}\text { Heat lift } \\
\text { Duty cycle }\end{array}$ & $\begin{array}{l}<1 \mu \mathrm{W} \\
90 \%\end{array}$ & $\begin{array}{l}>1 \mu \mathrm{W} \\
100 \%\end{array}$ & 2011 & 2015 \\
\hline $\begin{array}{l}\text { IXO } \\
\text { Push }\end{array}$ & $\begin{array}{l}\text { Large X-ray mirror } \\
\text { systems }\end{array}$ & $\begin{array}{l}\text { Effective Area } \\
\text { HPD Resolution } \\
\text { Areal Density; Active }\end{array}$ & $\begin{array}{l}0.3 \mathrm{~m} 2 \\
15 \operatorname{arcsec} \\
10 \mathrm{~kg} / \mathrm{m} 2 ; \text { no }\end{array}$ & $\begin{array}{l}>3 \mathrm{~m} 2(50 \mathrm{~m} 2) \\
<5 \operatorname{arcsec}(<1 \text { as }) \\
1 \mathrm{~kg} / \mathrm{m} 2 ; \text { yes }\end{array}$ & 2011 & $\begin{array}{c}2020 \\
(30)\end{array}$ \\
\hline $\begin{array}{l}\text { NWTP } \\
\text { UVOTP } \\
\text { Push }\end{array}$ & $\begin{array}{l}\text { Large UVOIR mirror } \\
\text { systems }\end{array}$ & $\begin{array}{l}\text { Aperture diameter } \\
\text { Figure } \\
\text { Stability } \\
\text { Reflectivity } \\
\mathrm{kg} / \mathrm{m} 2 \\
\$ / \mathrm{m} 2\end{array}$ & $\begin{array}{l}2.4 \mathrm{~m} \\
<10 \mathrm{~nm} \mathrm{rms} \\
--- \\
>60 \%, 120-900 \mathrm{~nm} \\
30 \mathrm{~kg} / \mathrm{m} 2 \\
\$ 12 \mathrm{M} / \mathrm{m} 2\end{array}$ & $\begin{array}{l}3 \text { to } 8 \mathrm{~m}(15 \text { to } 30 \mathrm{~m}) \\
<10 \mathrm{~nm} \mathrm{rms} \\
>9,000 \mathrm{~min} \\
>60 \%, 90-1100 \mathrm{~nm} \\
\text { Depends on } \mathrm{LV} \\
<\$ 1 \mathrm{M} / \mathrm{m} 2\end{array}$ & 2011 & $\begin{array}{c}2020 \\
(30)\end{array}$ \\
\hline WFIRST & Passive stable structure & Thermal stability & Chandra & WFOV PSF Stable & 2011 & 2014 \\
\hline NWTP & Large structure: occulter & Dia; Petal Edge Tol & Not demonstrated & $30-80 \mathrm{~m} ;<0.1 \mathrm{~mm} \mathrm{rms}$ & 2011 & 2016 \\
\hline $\begin{array}{l}\text { NWTP } \\
\text { UVOTP } \\
\text { Push }\end{array}$ & $\begin{array}{l}\text { Large, stable telescope } \\
\text { structures } \\
\text { (Passive or active) }\end{array}$ & $\begin{array}{l}\text { Aperture diameter } \\
\text { Thermal/dynamic WFE } \\
\text { Line-of-sight jitter } \\
\mathrm{kg} / \mathrm{m} 2 \\
\$ / \mathrm{m} 2\end{array}$ & $\begin{array}{l}6.5 \mathrm{~m} \\
60 \mathrm{~nm} \mathrm{rms} \\
1.6 \mathrm{mas} \\
40 \mathrm{~kg} / \mathrm{m} 2 \\
\$ 4 \mathrm{M} / \mathrm{m} 2\end{array}$ & $\begin{array}{l}8 \mathrm{~m}(15 \text { to } 30 \mathrm{~m}) \\
<0.1 \mathrm{~nm} \mathrm{rms} \\
1 \mathrm{mas} \\
<20(\text { or } 400) \mathrm{kg} / \mathrm{m} 2 \\
<\$ 2 \mathrm{M} / \mathrm{m} 2\end{array}$ & 2011 & $\begin{array}{l}2020 \\
(30)\end{array}$ \\
\hline $\begin{array}{l}\text { LISA } \\
\text { NWTP }\end{array}$ & $\begin{array}{l}\text { Drag-Free Flying } \\
\text { Occulter Flying }\end{array}$ & $\begin{array}{l}\text { Residual accel } \\
\text { Range } \\
\text { Lateral alignment }\end{array}$ & $3 \times 10^{-14} \mathrm{~m} / \mathrm{s}^{2} / \sqrt{\mathrm{Hz}}$ & $\begin{array}{l}3 \times 10^{-15} \mathrm{~m} / \mathrm{s}^{2} / \sqrt{\mathrm{Hz}} \\
10,000 \text { to } 80,000 \mathrm{~km} \\
\pm 0.7 \mathrm{~m} \text { wrt LOS }\end{array}$ & 2011 & 2016 \\
\hline $\begin{array}{l}\text { NWTP } \\
\text { Push }\end{array}$ & $\begin{array}{l}\text { Formation flying: } \\
\text { Sparse \& Interferometer }\end{array}$ & $\begin{array}{l}\text { Position/pointing } \\
\text { \#; Separation }\end{array}$ & $\begin{array}{l}5 \mathrm{~cm} / 6.7 \operatorname{arcmin} \\
2 ; 2 ; 2 \mathrm{~m}\end{array}$ & $5 ; 15-400-\mathrm{m}$ & 2011 & 2020 \\
\hline $\begin{array}{l}\text { LISA } \\
\text { Push }\end{array}$ & $\begin{array}{l}\text { Gravity wave sensor } \\
\text { Atomic interferometer }\end{array}$ & $\begin{array}{l}\text { Spacetime Strain } \\
\text { Bandpass }\end{array}$ & N/A & $\begin{array}{l}1 \times 10^{-21} / \sqrt{\mathrm{Hz}}, 0.1- \\
100 \mathrm{mHZ}\end{array}$ & 2013 & 2019 \\
\hline Various & Communication & Bits per sec & & Terra bps & & 2014 \\
\hline
\end{tabular}




\section{Astrophysics Technology Needs}

Astrophysics requires advancements in 5 SIOSS areas:

Detectors and electronics for X-ray and UV/optical/infrared (UVOIR);

Optical components and systems for starlight suppression, wavefront control, and enhanced UVOIR performance;

Low-power sub-10K cryo-coolers;

Large X-ray and UVOIR mirror systems (structures); and

Multi-spacecraft formation flying, navigation, and control.

Additionally, Astrophysics missions require other technologies:

Affordable volume and mass capacities of launch vehicles to enable largeaperture observatories and mid-capacity missions;

Terabit communication; and

Micro-Newton thrusters for precision pointing \& formation-flying control 


\section{Technology Area Breakdown Structure (TABS)}

Technology needs for each SMD area were deconstructed into broad categories.

For example, many missions require new or improved detectors.

These broad categories were condensed into 3 groups:

Remote Sensing Instruments/Sensors,

Observatories, and

In-situ Instruments/Sensors.

and organized into a 4-level TABS. 


\section{TA8: Technology Area Breakdown Structure}

\subsection{Science Instruments, Observatories \& Sensor Systems}

8.1 Remote Sensing

Instruments/Sensors

\section{(8.1.1)}

Detectors and Focal Planes

8.1.1.1 Large Format Arrays

8.1.1.2 Spectral Detectors

8.1.1.3 Polarization Sensitive Det.

8.1.1.4 Photon-Counting Det.

8.1.1.5 Radiation-Hardened Det.

8.1.1.6 Sub-Kelvin High-Sensitivity Det

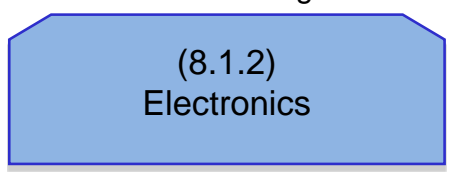

8.1.2.1 Radiation Hardened

8.1.2.2 Low Noise

8.1.2.3 High Speed

\section{(8.1.3)}

Optical Components

8.1.3.1 Starlight Suppression

8.1.3.2 Active Wavefront control

8.1.3.3 Optical Components

8.1.3.4 Advanced Spectrometers/Instruments

\section{(8.1.4)}

Microwave \& Radio Transmitters \& Receivers

8.1.4.1 Integrated Radar T/R Modules

8.1.4.2 Integrated Radiometer Receivers

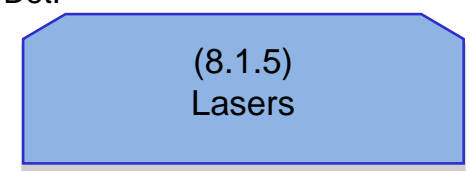

8.1.5.1 Pulsed Lasers

8.1.5.2 CW Lasers

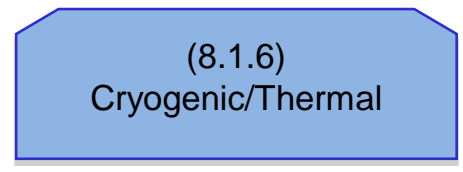

8.1.6.14-20K Cryo-Coolers for Space 8.1.6.2 Sub-Kelvin Coolers

\subsection{Observatories}

(8.2.1)

Large Mirror Systems

8.2.1.1 Grazing Incidence

8.2.1.2 Normal Incidence

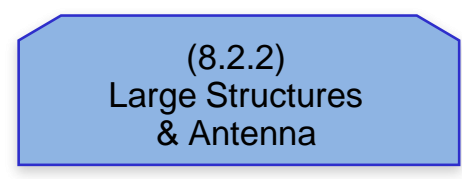

8.2.2.1 Passive Ultra-Stable Structures 8.2.2.2 Deployable/Assembled Tel. Support Structure and Antenna 8.2.2.3 Active Control

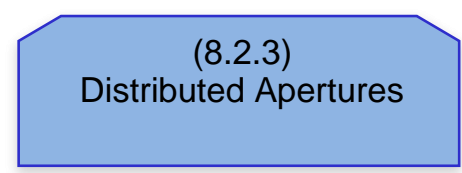

8.2.3.1 Formation Flying
8.3 In-Situ

Instruments/Sensors

\section{(8.3.1) \\ Particles}

8.3.1.1 Energetic Particle Det. (>30keV-NMeV)

8.3.1.2 Plasma Det. ( $<1 \mathrm{eV}-30 \mathrm{keV})$ 8.3.1.3 Magnetometers (DC \& $\mathrm{AC)}$

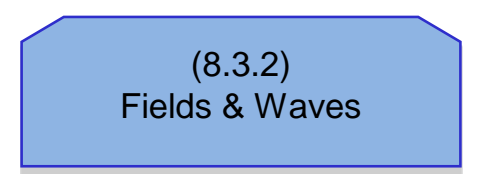

8.3.2.1 EM Field Sensors 8.3.2.2 Gravity-Wave Sensors

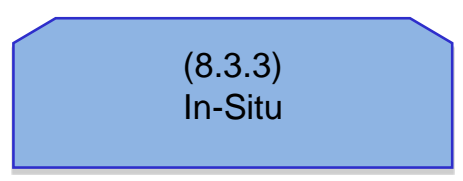

8.3.4.1 Sample Handling, Preparation, and Containment

8.3.4.2 Chemical and Mineral Assessment 8.3.4.3 Organic Assessment

8.3.4.4 Biological Detection \& Characterization 8.3.4.5 Planetary Protection 


\section{Technology Area Breakdown Structure (TABS)}

\section{Remote Sensing Instruments/Sensors:}

convert electromagnetic radiation (photons or waves) into science data or generate electromagnetic radiation (photons or waves);

typically require an observatory;

may be stand-alone sharing a common spacecraft bus

Observatory: collect, concentrate, and/or transmit photons.

In-situ Instruments/Sensors create science data from:

fields or waves (AC/DC electromagnetic, gravity, acoustic, seismic, etc); particles (charged, neutral, dust, etc.); or physical samples (chemical, biological, etc.). 


\section{Technology Development Roadmaps}

Development Roadmaps were developed for each SMD Division.

Roadmaps use TABS structure with direct traceability to identified mission needs for each Division.

Each technology need has specific maturity milestones (TRL-6).

Some technology needs have alternative pathway decision points.

Roadmaps explicitly includes 2020 \& 2030 Decadal Reviews

Explorer missions do not have explicit technology needs. 


\section{Astrophysics Technology Development Roadmap}

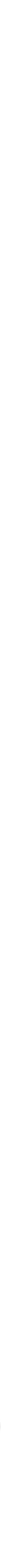




\section{Top Technical Challenges}

Top Challenges list was condensed from SMD assessments.

For near- \& mid-term investments, goal is to advance state of art for each Challenge by 2 to $10 \mathrm{X}$.

Long-term goal is to develop revolutionary capabilities

Investment must be balanced between short- and long-term to account for differences in maturity rates.

Top Technical Categories are not in any priority order; rather the list is organized by general need within selected timeframes.

Actual funding decisions will be determined by open competition and peer review. Competition is the fastest, most economical way to advance the state of the art. 


\section{Top Technical Challenges}

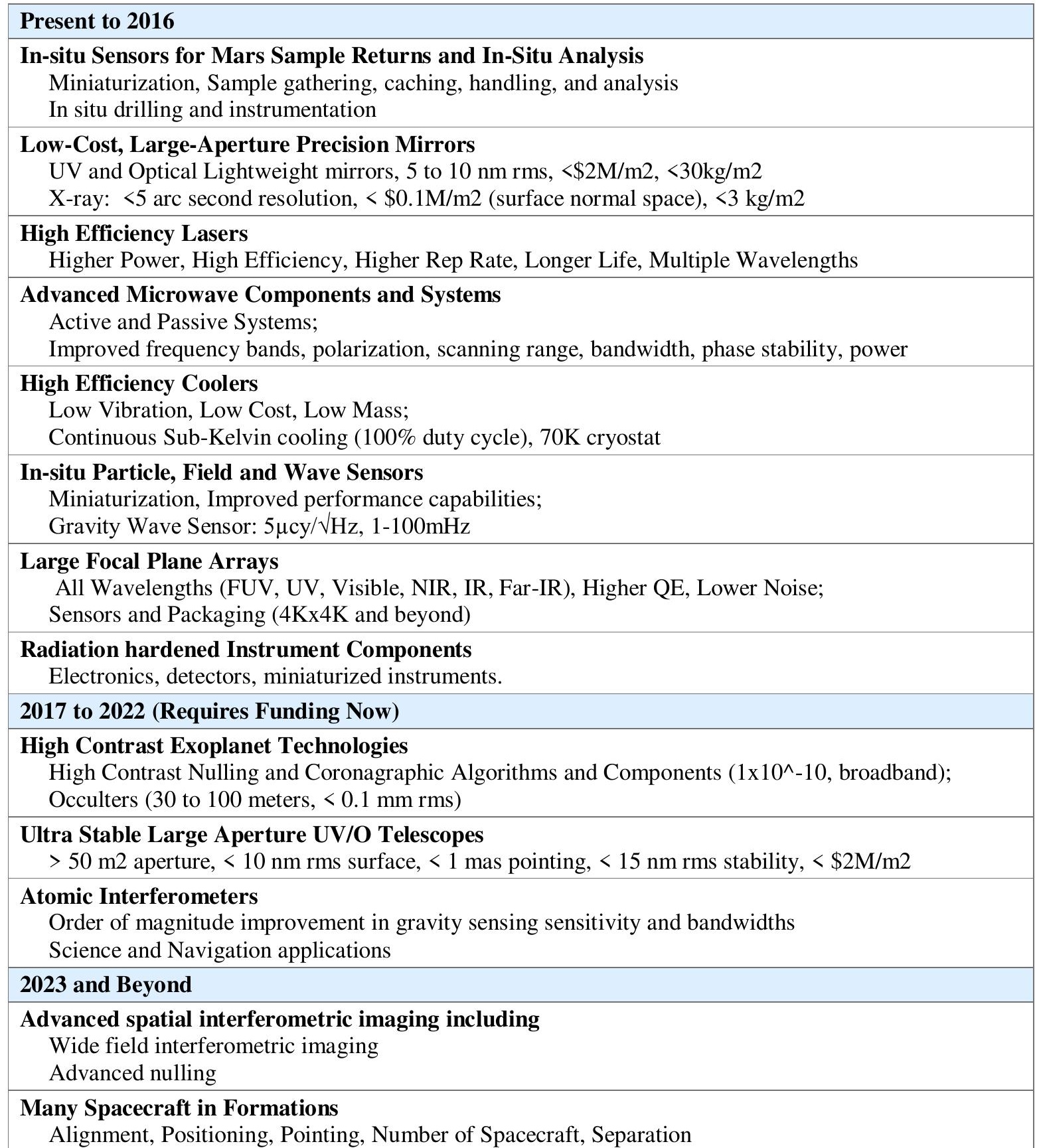




\section{Interdependencies with other Technology Areas}

Each TA identifies whether

Its Technology is Required by another TA

It Needs Technology from another Area

Technology flows both ways between Tas

SIOSS Technology flows both ways with all other TAs

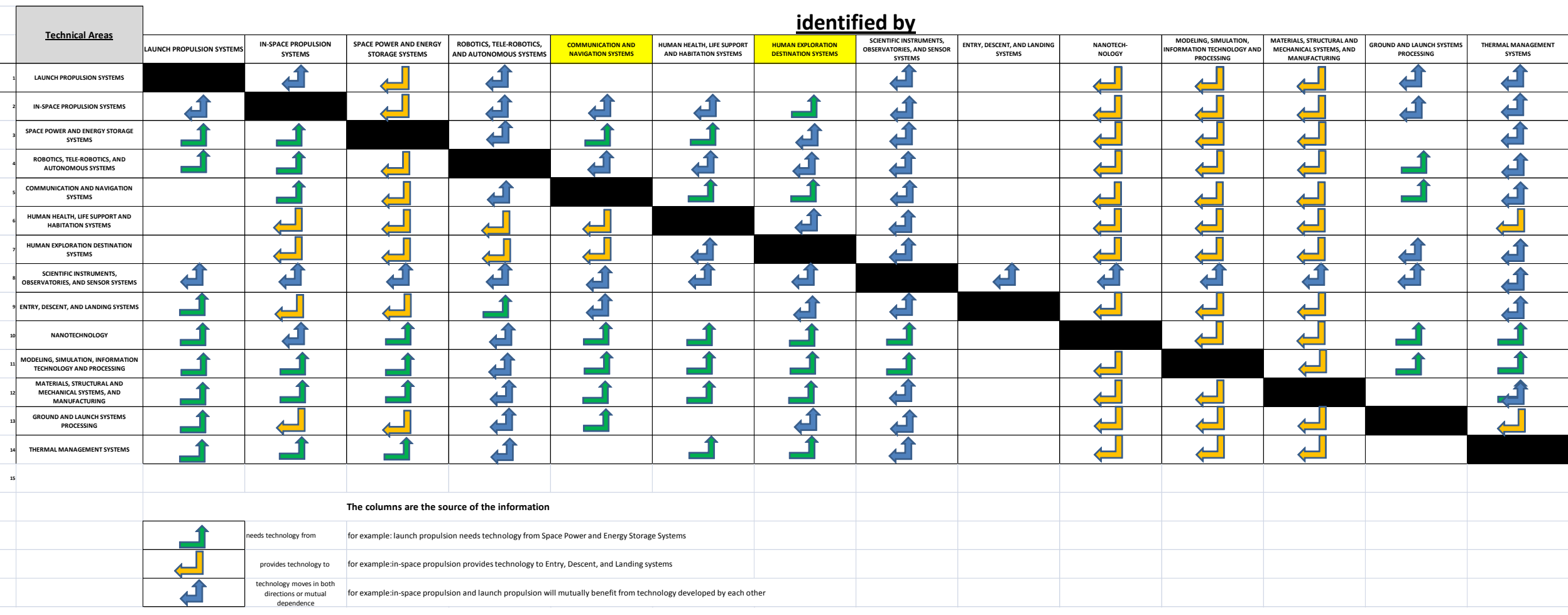




\section{Interdependencies with other Technology Areas}

\section{SIOSS technologies have interdependencies with all areas}

long-lived high-power lasers and single photon detectors for optical communication; large aperture solar concentrators for space power \& solar thermal propulsions; machine vision systems to aid human \& autonomous operations ranging from the assembly of flight hardware to AR\&D to 3D terrain descent imaging;

sub-20K cryo-coolers for infrared to far-infrared optical systems and detectors.

\begin{tabular}{|c|c|c|}
\hline \multicolumn{3}{|c|}{ Table 3-1 Interdependencies between SIOSS Technology and other Technology Areas } \\
\hline Technology Area & Other TA Technology required by SIOSS & SIOSS Technology required by Other TA \\
\hline TA1: Launch Propulsion & Affordable access to space, Heavy lift vehicle (PUSH) & $\begin{array}{l}\text { Integrated Health Monitoring (IHM) Sensors, Wireless } \\
\text { communication source/receiver }\end{array}$ \\
\hline TA2: In-Space Propulsion & Electric/ion propulsion, Micro-Newton thrusters, Solar sails, solar electric & $\begin{array}{l}\text { IHM Sensors, Solar Power, High Power Lasers, Tracking \& } \\
\text { Pointing }\end{array}$ \\
\hline TA3: Space Power \& Storage & Radioisotopes, L2 Power Grid (PUSH) & Photovoltaic Power, Laser Power Beaming, \\
\hline TA4: Robotics & $\begin{array}{l}\text { Rovers, sample acquisition \& containment, Aerobots, AR\&D; Robotic } \\
\text { servicing (PUSH), Robotic assembly (PUSH) }\end{array}$ & $\begin{array}{l}\text { Machine Vision; State Sensors, proximity, tactile; avoidance; } \\
\text { telepresence; active ranging }\end{array}$ \\
\hline TA5: Com \& Nav & $\begin{array}{l}\text { Terabit communication; Space Position System; Precision Formation } \\
\text { Flying (PUSH) }\end{array}$ & $\begin{array}{l}\text { Optical Communication; Precision Positioning \& Laser Ranging; } \\
\text { AR\&D sensors; Star Trackers; XNAV; Quantum Communication }\end{array}$ \\
\hline TA6: Human HAB & Human in-space assembly and service; Human Surface Science (PUSH) & $\begin{array}{l}\text { Crew-Protection Sensors; Crew Health Sensors; Space Weather } \\
\text { Sensors }\end{array}$ \\
\hline TA7: Human Exploration & $\begin{array}{l}\text { Heavy lift vehicle (PUSH); Human in-space assembly and servicing } \\
\text { (PUSH) }\end{array}$ & $\begin{array}{l}\text { Telescopes to survey NEO population; Instruments for missions to } \\
\text { NEOs \& other destinations (Moon, Mars, etc.); IHM sensors for } \\
\text { spacesuits; High-strength lightweight windows; solar concentrators }\end{array}$ \\
\hline $\begin{array}{l}\text { TA9: Entry, Descent \& } \\
\text { Landing }\end{array}$ & Planetary Descent Systems, Landers, Robots, Airships; Thermal Protection & $\begin{array}{l}\text { Terrain tracking and hazard avoidance sensors; IHM Sensors; } \\
\text { Planetary atmospheric characterization sensors }\end{array}$ \\
\hline TA10: Nano-Technology & $\begin{array}{l}\text { Sensors for chemical/bio assessment; High-strength, lightweight, CTE } \\
\text { materials; low-power radiation/fault tolerant electronics; nano-lasers; } \\
\text { miniaturized instruments; micro-fluidic labs on chip; single-photon } \\
\text { counting sensors; nano-thrusters for formation flying }\end{array}$ & Nanodevices are produced using optical lithographic methods \\
\hline TA11: Modeling & $\begin{array}{l}\text { Validated integrated performance modeling \& model-based systems } \\
\text { engineering }\end{array}$ & Validation Data Sensors \\
\hline $\begin{array}{l}\text { TA12: Materials \& } \\
\text { Structures }\end{array}$ & $\begin{array}{l}\text { Low-density, high stiffness, low-CTE materials for large, deployable or } \\
\text { assembly, active or passive, ultra-stiff/stable, precision structures (PUSH) }\end{array}$ & $\begin{array}{l}\text { IHM systems; NDE systems; dimensional and positional } \\
\text { characterization; Habitat Windows }\end{array}$ \\
\hline TA13: Ground/Launch Sys & Ability to integrate very large science missions & $\begin{array}{l}\text { IHM systems; corrosion detection; anomalous conditions } \\
\text { monitoring; NDE systems; Communication }\end{array}$ \\
\hline TA14: Thermal Management & Sub-20K Cryo-Coolers, Low-Power Cryocoolers & Optical emissivity coatings \\
\hline
\end{tabular}




\section{Benefits to Other National Needs}

SIOSS Technologies have potential benefit for a wide range of national needs, organizations and agencies:

- National Atmospheric and Oceanic Administration (NOAA)

- Department of Defense (DoD)

- Commercial Space Imaging Companies

- Department of Homeland Security (DHS)

- Department of Energy

- Department of Health and Human Services

- Food and Drug Administration

- Environmental Protection Agency 


\section{Benefits to Other National Needs}

Detectors/Focal Planes

Light-weight, small-size, low-power surveillance and night vision cameras Imaging Spectroscopy (aka Hyperspectral) Systems

Remote precision thermometry for surface-activity and energy-use sensing

Remote detection, identification, and quantification of gases

Micro/Radio transmit/receive (T/R) technologies

Dept. of Homeland Security detection systems, extending to THz systems

Lasers

Remote sensing of surface properties

High-bandwidth communications

Cryocoolers

Terrestrial precision metrology, quantum instruments

Mirrors/optics

Segmented Mirrors; Space Reconnaissance

Structures and Antennas

Synthetic and distributed aperture antennas

Particle, Fields, and Waves

Radiation detectors

In-Situ (unattended monitoring)

Toxic-substance monitors; Lab-on-a-chip applications 


\section{Public Input}

The National Research Council received 63 SIOSS inputs.

$\begin{array}{ll}67 \%(42 / 63) & 8.1 \text { Remote Sensing Instruments/Sensors } \\ 14 \%(9 / 63) & 8.2 \text { Observatories } \\ 19 \%(12 / 63) & 8.3 \text { In-Situ Instruments/Sensors }\end{array}$

Most were corrections, clarifications \& amplifications of content already in the report.

Others pointed out technologies which the assessment team had missed - such as needs for Gamma Ray science.

Many were made 'collective' or 'consensus' inputs on behalf of individual science communities. 


\section{Public Input}

8.1 Remote Sensing Instruments/Sensors

14 inputs regarding Detectors and Focal Planes

14 inputs regarding Electronics

9 inputs regarding Optical Components

3 input regarding Radio/Microwave;

1 input each regarding Lasers and Cryogenic/Thermal.

8.2 Observatories:

4 inputs regarding mirrors, antenna, coating

4 inputs regarding structures

1 input regarding formation flying

8.3 In-Situ Instruments/Sensors

5 inputs regarding gravity wave detection

4 inputs regarding atomic clocks

1 input each for neutral ion detection, quantum communication, mineral testing 


\section{Astrophysics Budget Planning}

The Decadal Survey recommended technology funding for:

1) Future missions at a level of $\sim 10 \%$ of NASA's anticipated budget for each mission to reduce risk and cost;

2) New Worlds, Inflation Probe and Future UV-Optical Space Capability Definition Technology Programs to prepare for missions beyond 2020; and

3) "General" technology to define, mature, and select approaches for future competed missions, and "Blue sky" technology to provide transformational improvements in capability and enable undreamed of missions. 


\section{Astrophysics Budget Planning}

Recommended Program and Technology Development

\begin{tabular}{|c|c|c|c|}
\hline Program & 10 -yr Total & $\underline{2012}$ & $\underline{2021}$ \\
\hline IXO & $\$ 200 \mathrm{M}$ & $\$ 4 \mathrm{M} / \mathrm{yr}$ & $\$ 30 \mathrm{M} / \mathrm{yr}$ \\
\hline Inflation Probe & $\$ 60$ to $\$ 200 \mathrm{M}$ & $\$ 4 \mathrm{M} / \mathrm{yr}$ & $\$ 30 \mathrm{M} / \mathrm{yr}$ \\
\hline New Worlds & $\$ 100$ to $\$ 200 \mathrm{M}$ & $\$ 4 \mathrm{M} / \mathrm{yr}$ & $\$ 30 \mathrm{M} / \mathrm{yr}$ \\
\hline UV-Optical & $\$ 40 \mathrm{M}$ & $\$ 2 \mathrm{M} / \mathrm{yr}$ & $\$ 10 \mathrm{M} / \mathrm{yr}$ \\
\hline
\end{tabular}

Recommended Augmentations to current \$40M/yr Investment Advanced Tech $\quad \$ 5 \mathrm{M} / \mathrm{yr}$

APRA $\$ 20 \mathrm{M}$ (25\% increase)

Intermediate Tech $\$ 100 \mathrm{M}(\$ 2 \mathrm{M} / \mathrm{yr}$ now to $\$ 15 \mathrm{M} / \mathrm{yr}$ by 2021$)$

10 -yr Total is $\$ 1$ to $\$ 1.2 \mathrm{~B}$ for TA8 SIOSS

This Total should be split primarily between TABS 8.1 Science Instruments and TABS 8.2 Observatory.

Astrophysics has limited TABLS 8.3 Sensor Systems needs. 


\section{Astrophysics Budget Planning}

Decadal recommended a 10-yr Budget of $\$ 1 \mathrm{~B}$ to $\$ 1.2 \mathrm{~B}$

Assuming that all Decadal Recommendations are for External

Funding, it is necessary to also define a NASA internal budget.

Assume NASA Internal Funding $=50 \%$ of External Funding

Allocated $75 \%$ of NASA Funding to Labor

Allocated $25 \%$ of NASA Funding to ODC

Thus $\$ 60 \mathrm{M} / \mathrm{yr}=$ approx 200 FTEs/yr and \$15M/yr ODC

This gives a Total TA8 SIOSS 10-ry Budget of $\$ 1.5 \mathrm{~B}$ to $\$ 1.8 \mathrm{~B}$ just to support the needs of Astrophysics, for example:
8.1 Science Instruments
$\$ 800 \mathrm{M}$
8.2 Observatory
$\$ 600 \mathrm{M}$
8.3 Sensor Systems
$\$ 200 \mathrm{M}$ 


\section{Decadal Analysis}

Similar analysis is required for the other Science Mission

Directorate Decadal Reports:

Earth Science

Heliophysics

Planetary 


\section{Conclusion}

Technology advancement is required to enable NASA's high priority missions of the future.

To prepare for those missions requires a roadmap of how to get from the current state of the art to where technology needs to be in $5,10,15$ and 20 years.

SIOSS identifies where substantial enhancements in mission capabilities are needed and provides strategic guidance for the agency's budget formulation and prioritization process.

The initial report was presented to the NRC in Oct 2010 (http://www.nasa.gov/offices/oct/home/roadmaps/index.html). And, the NRC review report is expected in late summer 2011. 
BACKUP 


\section{Earth Science Technology Needs}

Earth Science requires 4 areas:

- Advance antennas, receivers, transmitters, signal- and data-processing electronics, and cryo coolers.

- Improve low-areal density telescopes in the 1$m$ range, filters and coatings; advance low noise/highly efficient detectors, and focal planes with readout integrated circuits (ROIC); complementary detector arrays, electronics, cryo coolers and data processing systems and passive hyperspectral/multispectral/imagers, (UV-VisIR-FIR) and spectrometers (0.3 to $50 \mu \mathrm{m})$

- Advance lasers in 0.3-2.0 $\mu \mathrm{m}$ range (high power, multi-beam/multi-wavelength, pulsed, and continuous wave), detectors, receivers, larger collecting optics, and scanning mechanisms (including pointing and scanning at high angular resolution); improved quantum efficiency detectors, long-life, high-power laser diode arrays; high damage threshold optics

- Large telescope and RF antenna enable future climate and weather applications.

\begin{tabular}{|c|c|c|c|c|c|c|}
\hline Mission & Technology & \begin{tabular}{|l|} 
Metric \\
\end{tabular} & State of Art & Need & Start & TRL6 \\
\hline \multirow[t]{5}{*}{ ASCENDS } & $\begin{array}{l}\text { Multi-freq laser } \\
0.765 / 1.572 / 2.05 \\
\mu \mathrm{m} \text { Pulsed }\end{array}$ & \begin{tabular}{|l} 
Output energy \\
Rep rate \\
Efficiency \\
\end{tabular} & $\begin{array}{l}25 \mu \mathrm{J} / 25 \mu \mathrm{J} / 30 \mathrm{~mJ} \\
10 \mathrm{kHz} / 50 \mathrm{~Hz} \\
<2 / 4 \%\end{array}$ & $\begin{array}{l}>3 / 3 / 65 \mathrm{~mJ} \\
10 \mathrm{kHz} / 10 \mathrm{kHz} / 50 \mathrm{~Hz} \\
3.5 / 7 / 10 \%\end{array}$ & 2012 & 2014 \\
\hline & $1.6 \mu \mathrm{m} \mathrm{CW}$ laser & Power/module/efficiency & $5 \mathrm{~W} / 7 / 8 \%$ & $35 \mathrm{~W} / 1 / 10 \%$ & 2012 & 2014 \\
\hline & $1.26 \mu \mathrm{m} \mathrm{CW}$ laser & \begin{tabular}{|l|} 
Power/module/efficiency \\
\end{tabular} & $4 \mathrm{~W} / 1 / 3 \%$ & $20 \mathrm{~W} / 1 / 8 \%$ & 2012 & 2014 \\
\hline & $1.57 \mu \mathrm{m}$ detector & QE/gain/bandwidth & & $10 \% / 300 / 10 \mathrm{MHz}$ & 2012 & 2015 \\
\hline & $2 \mu \mathrm{m}$ APD detector & $\begin{array}{l}\text { QE/Bandwidth } \\
\text { NEP } \\
\end{array}$ & $\begin{array}{l}>55 \% / 10 \mathrm{MHz} \\
10^{-11} \mathrm{~W} / \mathrm{Hz}^{1 / 2}\end{array}$ & $\begin{array}{l}>55 \% />500 \mathrm{MHz} \\
10^{-14} \mathrm{~W}^{1-\mathrm{Hz}^{1 / 2}}\end{array}$ & 2012 & 2014 \\
\hline \multirow[t]{3}{*}{ SWOT } & $\begin{array}{l}\text { Ka-band power } \\
\text { switch matrix }\end{array}$ & Power capacity & $\sim 500 \mathrm{~W}$ peak & $\begin{array}{l}2.5 \mathrm{~kW} \text { peak, } 110- \\
165 \mathrm{~W} \text { avg.; Stable }\end{array}$ & 2012 & 2015 \\
\hline & Ka-band receiver & $\begin{array}{l}\text { Phase stability, isolation } \\
\text { Bandwidth }\end{array}$ & $\begin{array}{l}\sim 50 \mathrm{mdeg}, 68 \mathrm{~dB}, \\
80 \mathrm{MHz}\end{array}$ & $\begin{array}{l}-40 \mathrm{mdeg} \text { over } 3 \mathrm{~min}, \\
>80 \mathrm{~dB},>200 \mathrm{MHz}\end{array}$ & 2012 & 2015 \\
\hline & $\begin{array}{l}\text { Deployable-antenna } \\
\text { structure }\end{array}$ & \begin{tabular}{|l|} 
Boom length \\
Pointing stability \\
\end{tabular} & $\begin{array}{l}6.5 \mathrm{~m} \\
\sim 0.05 \text { arsec roll } \\
\end{array}$ & $\begin{array}{l}10-14 \mathrm{~m} \\
0.005 \mathrm{arcsec} \mathrm{roll} / 3 \mathrm{~min}\end{array}$ & 2012 & 2015 \\
\hline HyspIRI & $\begin{array}{l}\text { TIR spectrometer } \\
(8 \mathrm{ch}, 3-12 \mu \mathrm{m})\end{array}$ & Frame rate & $\sim 1$ Mpixels/sec & $\begin{array}{l}256 \mathrm{Mpixels} / \mathrm{sec} \text { at } \\
14 \mathrm{bits} ; 32 \mathrm{kHz}\end{array}$ & 2012 & 2016 \\
\hline $\begin{array}{l}\text { GEO- } \\
\text { CAPE }\end{array}$ & $\begin{array}{l}\begin{array}{l}\text { UV-Vis-NIR } \\
\text { spectrometer ROIC }\end{array} \\
\text { spte }\end{array}$ & $\begin{array}{l}\text { Size, pixel pitch, frame } \\
\text { rate, quantization, } \mathrm{QE} \\
\end{array}$ & & $\begin{array}{l}1024 \times 2048,<13 \mu \mathrm{m}, \\
4 \mathrm{MHz}, 16 \mathrm{bit},>60 \% \mathrm{uv}\end{array}$ & 2013 & 2019 \\
\hline \multirow[t]{5}{*}{$\mathrm{ACE}$} & $\begin{array}{l}\text { Damage-resistant } \\
\text { UV laser at } 355 \mathrm{~nm}\end{array}$ & $\begin{array}{l}\text { Energy, repetition rate } \\
\text { efficiency, lifetime }\end{array}$ & $250 \mathrm{~mJ} / 100 \mathrm{~Hz} / 5 \%$ & $\begin{array}{l}300 \mathrm{~mJ}, 100 \mathrm{~Hz}, \\
10 \%, 3-5 \mathrm{Yrs}\end{array}$ & 2012 & 2019 \\
\hline & $\begin{array}{l}\text { CCD Array (355/ } \\
532 \mathrm{~nm})\end{array}$ & QE, sampling rate & & $>70 \% / 90 \%,>5 \mathrm{MHz}$ & 2012 & 2019 \\
\hline & $\begin{array}{l}\text { Multi-angle } \\
\text { polarimeter ROIC }\end{array}$ & $\begin{array}{l}\text { High-processing speed @ } \\
\text { low noise }\end{array}$ & $\sim 100 \mathrm{kpix} / \mathrm{sec}$ & $\begin{array}{l}>10 \mathrm{Mpix} / \mathrm{sec} \\
<40 \text { electrons }\end{array}$ & 2012 & 2019 \\
\hline & $\begin{array}{l}\text { W-band radar } \\
\text { deployable antenna }\end{array}$ & $\begin{array}{l}\text { Reflector diameter } \\
\text { Surface accuracy }\end{array}$ & $1.5 \mathrm{~mm}$ rms@5 & $\begin{array}{l}\text { Main 5-6 m; sub4-5m } \\
<0.1 \mathrm{~mm} \text { RMS }\end{array}$ & 2013 & 2019 \\
\hline & $\begin{array}{l}\text { W/Ka-band dual- } \\
\text { freq. reflect array }\end{array}$ & \# Elements & & $\begin{array}{l}\text { W-band: } 2500 \\
\text { Ka-band: } 900\end{array}$ & 2013 & 2019 \\
\hline \multirow[t]{2}{*}{ LIST } & Photon-counting det & $\mathrm{QE}$ & $20 \%$ in a $4 \times 4$ arr & $50 \%$ in a $1 \times 1000$ arr & 2011 & 2018 \\
\hline & $\begin{array}{l}\text { Laser altimeter } \\
(1 \mu \mathrm{m})\end{array}$ & $\begin{array}{l}\text { Wallplug efficiency } \\
\text { Multi-beam array } \\
\text { PRF } \\
\end{array}$ & $\begin{array}{l}\sim 10 \% \\
9 @ 222 \mu \mathrm{J} / \text { beam }\end{array}$ & $\begin{array}{l}20 \% \\
1000 @ 100 \mu \mathrm{J} / \text { beam } \\
10 \mathrm{kHz}\end{array}$ & 2012 & 2018 \\
\hline \multirow[t]{2}{*}{ PATH } & $\begin{array}{l}\text { Correlator } \\
\end{array}$ & \begin{tabular}{|l|} 
Power level \\
\end{tabular} & $224 \mu \mathrm{W} @ 375 \mathrm{MHz}$ & $250 \mu \mathrm{W} @ 1 \mathrm{GHz}$ & 2014 & 2020 \\
\hline & $\begin{array}{l}\text { Low-mass, low- } \\
\text { noise receiver }\end{array}$ & $\begin{array}{l}\text { Noise level, power, } \\
\text { mass, frequencies }\end{array}$ & $500 \mathrm{~K}$ & $\begin{array}{l}400 \mathrm{~K},<50 \mathrm{~mW}, \\
<150 \mathrm{~g}, 60-183 \mathrm{GHz}\end{array}$ & 2014 & 2020 \\
\hline GRACE-2 & Accelerometer & \begin{tabular}{|l|} 
Acceleration accuracy \\
\end{tabular} & $1 \mathrm{e}-11 \mathrm{~m} / \mathrm{s} / \mathrm{s}$ & $<1 \mathrm{e}-12 \mathrm{~m} / \mathrm{s} / \mathrm{s}, 1-100 \mathrm{~s}$ & 2018 & 2021 \\
\hline SCLP & $\begin{array}{l}\text { Dual-polarized } \\
\text { multi-frequency } \\
\text { feed array }\end{array}$ & \begin{tabular}{|l|} 
Frequency bands \\
Polarization \\
Scanning range \\
\end{tabular} & & $\begin{array}{l}9.6 \text { to } 17.2 \mathrm{GHz} \\
\mathrm{H} \text { and } \mathrm{V} \text { for all freq } \\
>10-20 \text { degrees }\end{array}$ & 2017 & 2022 \\
\hline \multirow[t]{2}{*}{ GACM } & $\begin{array}{l}\text { Stable sub- } \mathrm{mm} \\
\text { scanning antenna }\end{array}$ & $\begin{array}{l}\text { Size, surface accuracy } \\
\text { Areal density } \\
\end{array}$ & $\begin{array}{l}1.8 \mathrm{~m}, 10 \mu \mathrm{m} \mathrm{rms} \\
10 \mathrm{~kg} / \mathrm{m}^{\wedge} 2\end{array}$ & $\begin{array}{l}4 \mathrm{~m}, 10 \mu \mathrm{m} \text { rms } \\
<10 \mathrm{~kg} / \mathrm{m}^{\wedge} 2\end{array}$ & 2015 & 2023 \\
\hline & $\begin{array}{l}\text { Radiation-tolerant, } \\
\text { digital spectrometer }\end{array}$ & $\begin{array}{l}\text { Bandwidth } \\
\text { Efficiency } \\
\text { Channels } \\
\end{array}$ & $\begin{array}{l}0.75 \mathrm{GHz} \\
6 \mathrm{~W} / \mathrm{GHz} \\
4000 \\
\end{array}$ & $\begin{array}{l}8 \mathrm{GHz} \\
<1.5 \mathrm{~W} / \mathrm{GHz} \\
8000 \\
\end{array}$ & 2018 & 2023 \\
\hline push & $\begin{array}{l}\text { UV laser at } 305- \\
308 \mathrm{~nm} / 320-325 \mathrm{~nm}\end{array}$ & Efficiency, Output Energy & $100 \mathrm{mj}$ & $50 \mathrm{mj}$ & 2012 & 2023 \\
\hline \multirow[t]{4}{*}{ 3-D Winds } & $\begin{array}{l}\text { Multi-freq laser } \\
-2 / 1 \mu \mathrm{m} \text { pulsed }\end{array}$ & $\begin{array}{l}\text { Output energy/rep rate/ } \\
\text { WPE/laser lifetime }\end{array}$ & $\begin{array}{l}250 / 5 \mathrm{~Hz} / 2 \% \text { at } \\
2 u m\end{array}$ & $\begin{array}{l}250 / 500 \mathrm{~mJ} / 5 / 200 \mathrm{~Hz} \\
5 \% / 12 \%, 500 \mathrm{M} / 15 \mathrm{~B} \\
\text { shots }\end{array}$ & 2014 & 2024 \\
\hline & $\begin{array}{l}-2 \mu \mathrm{m} \mathrm{CW} \mathrm{seed} \\
\text { laser }\end{array}$ & Power & $60 \mathrm{~mW}$ & $100 \mathrm{~mW}$ & 2014 & 2024 \\
\hline & $\begin{array}{l}\text { Damage-resistant } \\
355 \mathrm{~nm} \text { pulsed laser }\end{array}$ & $\begin{array}{l}\text { Output energy; pulse rep } \\
\text { rate; WPE; life }\end{array}$ & & $\begin{array}{l}320-32 \mathrm{~mJ} / \text { pulse; } 120- \\
1500 \mathrm{~Hz} ;>5 \% ; 3 \mathrm{yrs}\end{array}$ & 2014 & 2024 \\
\hline & Lightweight mirrors & Diameter; areal density & & $>0.7 \mathrm{~m} ;<6 \mathrm{~kg} / \mathrm{m}^{\wedge} 2$ & 2018 & 2024 \\
\hline
\end{tabular}




\section{Heliophysics Technology Needs}

\section{Heliophysics requires 5 areas:}

- UV and EUV detectors (sensitivity, solar blindness, array size, and pixel counts)

- Reduce noise and insensitivity of electronics and detectors to heat and radiation

- Improve UV and EUV optical components (coating reflectivity and polarization uniformity, grating efficiency, and surface figure quality)

- Improve cryo-coolers for IR detectors

- Improve in-situ particle sensoraperture size and composition identification.

\begin{tabular}{|c|c|c|c|c|c|c|}
\hline Mission & 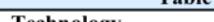 & & & & & \\
\hline $\begin{array}{l}\text { DGC } \\
\text { INCA } \\
\text { CISR }\end{array}$ & Pointing system & $\begin{array}{l}\text { Accuracy and } \\
\text { knowledge }\end{array}$ & $0.1 \mathrm{deg} / .05 \mathrm{deg}$ & $0.02 \mathrm{deg} / 0.02 \mathrm{deg}$ & 2013 & 2018 \\
\hline $\begin{array}{l}\text { DGC } \\
\text { ONEP }\end{array}$ & $\begin{array}{l}\text { Wide angle optical } \\
\text { reflective systems } \\
\text { Isolate } 83.4 \mathrm{~nm} \text { from } \\
121.6 \mathrm{~nm}\end{array}$ & $\begin{array}{l}\text { Wide FOV } \\
\text { Aperture } \\
\text { Spectral rejection of } \\
121.6 \text { and acceptance } \\
\text { of } 83.4 \mathrm{~nm}\end{array}$ & $\begin{array}{l}20 \mathrm{deg} \\
3 \mathrm{~cm} \\
1: 30\end{array}$ & $\begin{array}{l}30 \mathrm{deg}, \\
6 \text { to } 50 \mathrm{~cm} \\
1: 3000\end{array}$ & 2011 & 2014 \\
\hline $\begin{array}{l}\text { DGC } \\
\text { ONEP } \\
\text { INCA } \\
\text { CISR }\end{array}$ & $\begin{array}{l}\text { Spectral filters } \\
\text { Solar blind sensors } \\
\text { FUV sensors }\end{array}$ & $\begin{array}{l}\text { Resolution } \\
\text { Reflectivity in } 60-200 \\
\mathrm{~nm} \text {; Rejection } \\
\text { QE } 60-200 \mathrm{~nm}\end{array}$ & $\begin{array}{l}5 \mathrm{~nm} \text { FWHM } \\
80 \% \\
10 \mathrm{e}-6 \\
20 \% \\
\end{array}$ & $\begin{array}{l}2 \mathrm{~nm} \text { FWHM } \\
>90 \% \\
10 \mathrm{e}-8 \\
>50 \% \\
\end{array}$ & 2011 & 2014 \\
\hline Push & Miniaturization & Mass and power & $15 \mathrm{~kg} / 10 \mathrm{~W}$ & $3 \mathrm{~kg} / 5 \mathrm{~W}$ & 2013 & 2016 \\
\hline $\begin{array}{l}\text { SEPAT } \\
\text { HMag } \\
\text { DGC }\end{array}$ & $\begin{array}{l}\text { Fast, low-noise, } \\
\text { Rad-hard O/UV } \\
\text { detector }\end{array}$ & $\begin{array}{l}\text { Pixel array, pixel rate, } \\
\text { Read noise, rad } \\
\text { tolerance }\end{array}$ & $\begin{array}{l}1 \mathrm{kx} 1 \mathrm{k}, 10 \\
\mathrm{MHz}, 100 \mathrm{e}- \\
50 \mathrm{krad}\end{array}$ & $\begin{array}{l}2 \mathrm{kx} 2 \mathrm{k}, 60 \mathrm{MHz} \\
20 \mathrm{e}, 200 \mathrm{krad}\end{array}$ & 2013 & 2016 \\
\hline GRIPS & $\begin{array}{l}70 \mathrm{~K} \text { cryostat } \\
\text { with many channels }\end{array}$ & $\begin{array}{l}\text { Number of channels } \\
\text { Thermal leakage }\end{array}$ & $\begin{array}{l}\sim 30, \\
\sim 10 \mathrm{~mW} / \mathrm{ch}\end{array}$ & $\begin{array}{l}\sim 5000, \\
<1 \mathrm{~mW} / \mathrm{ch} .\end{array}$ & 2011 & 2014 \\
\hline GRIPS & $\sim 20$-m boom & $\begin{array}{l}\text { Boom control, tip } \\
\text { mass }\end{array}$ & & $\sim 0.5 \mathrm{deg}, 50 \mathrm{~kg}$ & 2012 & 2014 \\
\hline Push & $\begin{array}{l}\text { Fast electronics } \\
\end{array}$ & $\begin{array}{l}\text { Timing } \\
\text { Dead time per event }\end{array}$ & $\begin{array}{l}10 \mathrm{~ns} \\
300 \mathrm{~ns}\end{array}$ & $\begin{array}{l}3 \mathrm{~ns} \\
\sim 30 \mathrm{~ns}\end{array}$ & 2012 & 2014 \\
\hline $\begin{array}{l}\text { ONEP } \\
\text { Push }\end{array}$ & $\begin{array}{l}2 \text { spacecraft } \\
\text { Formation flying }\end{array}$ & $\begin{array}{l}\text { Alignment } \\
\text { Aspect } \\
\text { Separation control }\end{array}$ & None & $\begin{array}{l}1 \text { arcsec } \\
0.1 \text { arcsec } \\
100 \pm 0.1 \mathrm{~m}\end{array}$ & 2011 & 2015 \\
\hline Push & $\mathrm{X}$-ray focusing lens & $\begin{array}{l}\text { Energy range } \\
\text { Angular resolution }\end{array}$ & $\begin{array}{l}-6 \mathrm{keV} \\
1 \mathrm{arcsec}\end{array}$ & $\begin{array}{l}1-20 \mathrm{keV} \\
<0.1 \mathrm{arcsec} \\
\end{array}$ & 2011 & 2014 \\
\hline FOXSI & $\begin{array}{l}\text { Hard X-ray focusing } \\
\text { mirrors }\end{array}$ & $\begin{array}{l}\text { Energy range } \\
\text { FWHM Resolution }\end{array}$ & $\begin{array}{l}5-30 \mathrm{keV} \\
<10 \text { arcsec }\end{array}$ & $\begin{array}{l}5-100 \mathrm{keV} \\
5 \operatorname{arcsec}\end{array}$ & 2011 & 2014 \\
\hline $\begin{array}{l}\text { Push } \\
\end{array}$ & X-ray polarization & $\begin{array}{l}\text { Energy range } \\
\text { Min. polarization }\end{array}$ & $\begin{array}{l}<10 \mathrm{keV} \\
10 \%\end{array}$ & $\begin{array}{l}\text { Up to } 50 \mathrm{keV} \\
1 \%\end{array}$ & 2011 & 2014 \\
\hline Push & $\begin{array}{l}\text { X-ray modulation } \\
\text { grids }\end{array}$ & $\begin{array}{l}\text { Finest pitch } \\
\text { No. of pitches per grid }\end{array}$ & $\begin{array}{l}34 \mu \mathrm{m} \\
16\end{array}$ & $\begin{array}{l}10 \mu \mathrm{m} \\
100\end{array}$ & 2011 & 2014 \\
\hline $\begin{array}{l}\text { Push } \\
\text { Pus }\end{array}$ & $\begin{array}{l}\text { X-ray TES } \\
\text { microcalorimeters }\end{array}$ & $\begin{array}{l}\text { Resolution, count } \\
\text { rate/pixel } \\
\text { Number of pixels, } \\
\text { Pixel packing }\end{array}$ & $\begin{array}{l}4 \mathrm{eV}, 300 \mathrm{c} / \mathrm{s}, \\
32 \times 32 \\
150 \times 150 \mu \mathrm{m}\end{array}$ & $\begin{array}{l}2 \mathrm{eV}, 1,000 \mathrm{c} / \mathrm{s}, \\
1000 \times 1000, \\
75 \times 75 \mu \mathrm{m}\end{array}$ & 2011 & 2015 \\
\hline Push & $\begin{array}{l}\text { Solid-state X-ray } \\
\text { detectors }\end{array}$ & $\begin{array}{l}\text { Counting rate } \\
\text { Pixel size }\end{array}$ & $\begin{array}{l}1000 \mathrm{c} / \mathrm{s} \\
500 \mu \mathrm{m}\end{array}$ & $\begin{array}{l}10,000 \mathrm{c} / \mathrm{s} \\
100 \mu \mathrm{m}\end{array}$ & 2011 & 2014 \\
\hline $\begin{array}{l}\text { Solar } \\
\text { CubeSat }\end{array}$ & $\begin{array}{l}\text { Deployable photon } \\
\text { sieve }\end{array}$ & $\begin{array}{l}\text { Diameter } \\
\text { Transmission } \\
\text { Optical resolution }\end{array}$ & $\begin{array}{l}30 \mathrm{~cm}, 1 \% \text {, } \\
0.5 \text { arcsec }\end{array}$ & $\begin{array}{l}2 \mathrm{~m},>5 \% \\
0.1 \operatorname{arcsec}\end{array}$ & 2012 & 2014 \\
\hline ONEP & $\geq 20 \mathrm{~m}$ Boom & Stiffness & & $10^{7} \mathrm{~N} \mathrm{~m}^{2}$ & 2012 & 2015 \\
\hline Push & UV image slicer & $\begin{array}{l}\text { Number of slices } \\
\text { Wavelength range }\end{array}$ & $\begin{array}{l}5 \\
>300 \mathrm{~nm} \\
\end{array}$ & $\begin{array}{l}20 \\
\text { Down to } 90 \mathrm{~nm}\end{array}$ & 2012 & 2014 \\
\hline ONEP & E-field boom & Length, mass & $10 \mathrm{~m}, 7 \mathrm{~kg}$ & $20 \mathrm{~m}, 4 \mathrm{~km}$ & 2012 & 2014 \\
\hline $\begin{array}{l}\text { ONEP } \\
\text { Various }\end{array}$ & $\begin{array}{l}\text { Electrostatically } \\
\text { clean solar array }\end{array}$ & $\begin{array}{l}\text { Power loss due to } \\
\text { cover and coating }\end{array}$ & $\begin{array}{l}20-25 \% \text { loss; } \\
\text { cost is \$Ms }\end{array}$ & $5 \%, \$ 500 \mathrm{~K}$ & 2011 & 2013 \\
\hline SEPAT & $\begin{array}{l}\text { Fast }(0.01 \mathrm{~s}) \text { imaging } \\
\text { electron } \\
\text { spectrometer }\end{array}$ & $\begin{array}{l}0.01 \mathrm{~s} \text { Static } 4 \mathrm{Pi} \mathrm{sr} \\
\text { FOV } / .01-2 \mathrm{keV} \text { with } \\
\text { static energy angle } \\
\text { analysis (SEAA) }\end{array}$ & $\begin{array}{l}0.5 \mathrm{~s}-\text { Top Hat } \\
\text { Energy-angle } \\
\text { analyzer (not } \\
\text { static) }\end{array}$ & $\begin{array}{l}0.01 \mathrm{~s} / \text { velocity distribut } \\
\text { SEAAs: } 4 \mathrm{Pi} \text { sr/ energy } \\
0.01-2 \mathrm{keV} / 7 \% \text { energy } \\
\text { resolution }\end{array}$ & 2011 & 2013 \\
\hline INCA & $\begin{array}{l}\text { WINCS: Wind Ion- } \\
\text { drift (temperatures) } \\
\text { Neutral/ion } \\
\text { Composition }\end{array}$ & $\begin{array}{l}\text { 1s cadence for } \\
\text { WINCS @ 400 km } \\
\text { altitude - 1W total } \\
\text { power }\end{array}$ & $\begin{array}{l}\text { Cross-track } \\
\text { component of } \\
\text { wind only @30 } \\
\text { W for all } \\
\text { measurements }\end{array}$ & $\begin{array}{l}\text { 1s cadence for Wind } \\
\text { I } \\
\text { IonDrift/Temp/Comp } \\
\text { @ } 400 \mathrm{~km} \text { altitude - } \\
1 \mathrm{~W} \text { total power with } \\
\text { onboard data analysis }\end{array}$ & 2013 & 2017 \\
\hline
\end{tabular}




\section{Planetary Science Technology Needs}

\section{Planetary Science needs:}

- Active spectroscopy and lasers

- Chemical and mineralogy assessment for Inner Planets missions

- Sample caching, handling and screening for Mars sample return

- Radiation-hardened electronics technology for Outer Planets missions

- Mass spectroscopy and organic detection technologies for missions to Saturn/Titan

- Sample gathering, handling and analysis for future Small Bodies mission.

\begin{tabular}{|c|c|c|c|c|c|c|}
\hline Mission & Technology & Metric & State of Art & Need & Start & TRL6 \\
\hline \multirow{7}{*}{$\begin{array}{l}\text { Discovery } \\
13 / 14, \\
\text { New } \\
\text { Frontiers 4, } \\
\text { EJSM }\end{array}$} & $\begin{array}{l}\text { Large arrays: Vis \& } \\
\text { IR }\end{array}$ & \begin{tabular}{|l|} 
Pixel count \\
\end{tabular} & $1 \mathrm{kx} 1 \mathrm{k}$ format & $>2 \mathrm{k} \times 2 \mathrm{k}$ format & 2011 & 2015 \\
\hline & Spectral-tunable IR & $\begin{array}{l}\text { Narrow-band// } \\
\text { range }\end{array}$ & $1 \mu \mathrm{m} /$ few $\mu \mathrm{m}$ & $0.1 \mu \mathrm{m} / 1-15 \mu \mathrm{m}$ & 2015 & 2018 \\
\hline & $\begin{array}{l}\text { Spectral-tune Sub- } \\
\mathrm{mm}\end{array}$ & $\begin{array}{l}\text { Tunability@ } \mathrm{x} \\
\text { GHz }\end{array}$ & $60 @ 600 \mathrm{GHz}$ & >150 GHz@1200 & 2015 & 2018 \\
\hline & $\begin{array}{l}\gamma \text {-ray, neutron } \\
\text { detectors }\end{array}$ & $\begin{array}{l}\text { Energy resolution, } \\
\text { Directionality }\end{array}$ & $1 \%, 10 \mathrm{deg}$ & $0.1 \%, 1 \mathrm{deg}$ & 2015 & 2018 \\
\hline & Polarization & $\begin{array}{l}\text { s/p, switching } \\
\text { speed }\end{array}$ & $50 \%, \sim 1 \mathrm{~Hz}$ & $>90 \%,>50 \mathrm{~Hz}$ & 2013 & 2018 \\
\hline & Photon Counting & $\Lambda$, array size & Some $\lambda$ 's: & UV/vis InGaAs & 2010 & 2018 \\
\hline & Rad hard Detector & TID, no SEU/SEL & Heavy shielding & $<100$ mils shield & 2010 & 2020 \\
\hline \multirow{3}{*}{$\begin{array}{l}\text { Dis } 13 / 14, \\
\text { NF } 4 \text {, } \\
\text { EJSM }\end{array}$} & Rad Hard Electronics & TID tolerance & $0.1-1 \mathrm{Mrad}$ & $3 \mathrm{Mrad}$ & 2010 & 2020 \\
\hline & $\begin{array}{l}\text { Low Noise } \\
\text { Electronics }\end{array}$ & \begin{tabular}{|l} 
Noise level (\%) \\
\end{tabular} & $<1 \%$ & $<0.01 \%$ & 2011 & 2020 \\
\hline & $\begin{array}{l}\text { Extreme Environment } \\
\text { Electronics }\end{array}$ & \begin{tabular}{|l|} 
Operating \\
temperature \\
\end{tabular} & $-55 \mathrm{C}$ to $125 \mathrm{C}$ & $-180 \mathrm{C}$ to $125 \mathrm{C}$ & 2011 & 2020 \\
\hline \multirow{2}{*}{$\begin{array}{l}\text { Dis 13/14, } \\
\text { NF 4, } \\
\text { Mars 2018, } \\
\text { EJSM } \\
\end{array}$} & $\begin{array}{l}\text { UV to Sub-mm Filters } \\
\text { \& Optical Coatings }\end{array}$ & $\begin{array}{l}\text { Transmission; } \\
\text { Uniform Polarize; } \\
\text { Band-pass }\end{array}$ & $\begin{array}{l}\text { T 90\%; } \\
\text { U 80\%; } \\
1 \mathrm{~nm}\end{array}$ & $\begin{array}{l}\mathrm{T}>97 \% \\
\mathrm{U}>90 \% \\
<1 \mathrm{~nm}\end{array}$ & 2012 & 2020 \\
\hline & Mini Spectrometer & \begin{tabular}{|l|} 
Mass \& Function \\
\end{tabular} & $5-10 \mathrm{~kg}$; Single & $1-3 \mathrm{~kg}$ & 2010 & 2020 \\
\hline \multirow[t]{2}{*}{$\begin{array}{l}\text { Dis } 13 / 14, \\
\text { NF } 4,\end{array}$} & $\begin{array}{l}\text { Integrated radar } \mathrm{T} / \mathrm{R} \\
\text { mods. }\end{array}$ & \begin{tabular}{|l}
$\begin{array}{l}\text { Power and } \\
\text { efficiency }\end{array}$ \\
\end{tabular} & $10-30 \mathrm{~W}, 40 \%$ & $10-30 \mathrm{~W}, 60 \%$ & 2013 & 2020 \\
\hline & $\begin{array}{l}\text { Integrated radiometer } \\
\text { receiver }\end{array}$ & \begin{tabular}{|l|} 
Size, Frequency, \\
Temp \\
\end{tabular} & $\begin{array}{l}100 \text {-ele; } 100 \mathrm{GHz}, \\
\text { Ambient Ops }\end{array}$ & $\begin{array}{l}\text { Quantum-limited; } \\
30-110 \mathrm{GHz} \text {; Cryo }\end{array}$ & 2013 & 2020 \\
\hline \multirow[t]{5}{*}{$\begin{array}{l}\text { Dis 13/14, } \\
\text { NF 4, } \\
\text { Mars 2018, } \\
\text { EJSM }\end{array}$} & $\begin{array}{l}\text { Pulsed lasers: } \\
\text { Altimeters, LIDAR }\end{array}$ & $\begin{array}{l}\text { Profiling, } \\
\text { lifetime, sampling } \\
\text { rate, } \\
\text { power } \\
\end{array}$ & $\begin{array}{l}\text { Single profiling, } \\
6 \times 10^{8} \text { shots, } 1-40 \\
\mathrm{~Hz} \\
200-10 \mathrm{~mJ} / \text { pulse }\end{array}$ & $\begin{array}{l}\text { Multi-beams, }>10^{9} \\
\text { shots, } 40-100 \mathrm{kHz} \\
300-0.3 \mathrm{~mJ} / \text { pulse }\end{array}$ & 2013 & 2020 \\
\hline & $\begin{array}{l}\text { Pulsed lasers: Raman, } \\
\text { LIBS }\end{array}$ & $\begin{array}{l}\text { Lifetime, } \\
\text { Sampling rate, } \\
\text { Power } \\
\end{array}$ & $\begin{array}{l}6 \times 10^{8} \text { shots, } 5 \mathrm{~Hz} \\
40 \mathrm{~mJ} / \text { pulse }\end{array}$ & $\begin{array}{l}>10^{9} \text { shots, }>10 \\
\mathrm{~Hz},>200 \mathrm{~mJ} / \text { pulse }\end{array}$ & 2013 & 2020 \\
\hline & $\mathrm{CW}$ lasers & $\begin{array}{l}\text { Peak power at } \\
<250 \mathrm{~nm}\end{array}$ & $10 \mathrm{~mW}$ & $>100 \mathrm{~mW}$ & 2013 & 2020 \\
\hline & $\mathrm{CW}$ tunable NIR/IR & $\begin{array}{l}\text { Room temp. } \\
\text { operation }\end{array}$ & Some $\lambda$ regions & $1-15 \mu \mathrm{m}$ & 2013 & 2020 \\
\hline & Diode lasers & $\begin{array}{l}\text { Power at } 1.083 \\
\mu \mathrm{m}\end{array}$ & $1 \mathrm{~mW}$ & $>10 \mathrm{~mW}$ & 2013 & 2020 \\
\hline \multirow{3}{*}{$\begin{array}{l}\text { Dis } 13 / 14, \\
\text { NF 4, } \\
\text { Mars 2018, } \\
\text { EJSM }\end{array}$} & Particle Detectors & Energy thresholds & $\begin{array}{l}\sim 10 \mathrm{keV} \text {, small } \\
\text { array }\end{array}$ & $\begin{array}{l}\sim 1 \mathrm{keV} \text {, large } \\
\text { array }\end{array}$ & 2013 & 2020 \\
\hline & Magnetometers & \begin{tabular}{|l|}
$\begin{array}{l}\text { Sensitive, boom } \\
\text { dist }\end{array}$ \\
\end{tabular} & $\begin{array}{l}\sim 10 \mathrm{pT} ; 3-10 \\
\text { meter }\end{array}$ & $\sim 1 \mathrm{pT} ;<1 \mathrm{~m}$ & 2013 & 2020 \\
\hline & EM Field Sensors & \begin{tabular}{|l} 
ADC; Coverage \\
\end{tabular} & 8-bit; limited & 18-bit; entire band & 2013 & 2020 \\
\hline \multirow{7}{*}{$\begin{array}{l}\text { Dis 13/14, } \\
\text { NF 4, } \\
\text { Mars 2018 } \\
\text { MSR }\end{array}$} & Gas composition & $\begin{array}{l}\text { Detection; } \\
\text { Precision } \\
\end{array}$ & $\begin{array}{l}\text { 1ppmv- } \\
\text { lppbv;10/mil }\end{array}$ & $0.01 \mathrm{ppbv} ; 0.1 / \mathrm{mil}$ & 2011 & 2020 \\
\hline & $\begin{array}{l}\text { Elemental } \\
\text { composition }\end{array}$ & Separation & $0.5 \mathrm{wt} \%$ & $0.1 \mathrm{wt} \%$ & 2011 & 2020 \\
\hline & $\begin{array}{l}\text { Mineral: APXS, IR, } \\
\gamma-, \text { Raman, XRD, } \\
\text { neutron }\end{array}$ & \begin{tabular}{|l} 
Detection limits \\
\end{tabular} & Few wt $\%$ & $<1 \mathrm{wt} \%$ & 2011 & 2020 \\
\hline & Age dating & \pm Myr error/Byr & $\pm 20 \mathrm{Myr}$ in lab & $\begin{array}{l} \pm 200 \mathrm{Myr} \text { on } \\
\text { surface }\end{array}$ & 2011 & 2020 \\
\hline & Biological & \begin{tabular}{|l|} 
Sensitivity \\
\end{tabular} & $\mathrm{Ppb}$ & ppt & 2011 & 2020 \\
\hline & Sample handing & $\%$ cross contam & $3-5 \%$ & $<0.1 \%$ & 2011 & 2020 \\
\hline & Instrument extreme & Temperature & -100 to $200 \mathrm{C}$ & -100 to $200 \mathrm{C}$ & 2011 & 2020 \\
\hline
\end{tabular}




\section{Technology Area 8.1 Science Instruments}

Major challenges include:

- Detectors/Focal Planes: Improve sensitivity and operating temp of single-element and large-array devices.

- Electronics: Radiation-hardened with reduced volume, mass and power.

\begin{tabular}{|c|c|c|c|c|c|}
\hline Technology & State of Art & Need & Start & TRL6 & $\begin{array}{l}\text { SMD } \\
\text { Division }\end{array}$ \\
\hline \multicolumn{6}{|c|}{ 8.1.1.1 Large Format Arrays } \\
\hline NIR \& TIR Detectors & $\begin{array}{l}\text { Pixel array: } 2 \mathrm{k} \mathrm{x} 2 \mathrm{k} \\
\text { Pixel size: } 18 \mu \mathrm{m}\end{array}$ & $\begin{array}{l}4 \mathrm{k} \times 4 \mathrm{k} \\
10 \mu \mathrm{m}\end{array}$ & 2011 & 2014 & Astro Earth \\
\hline $\begin{array}{l}\text { TIR Spectrometer } \\
\text { detectors } \\
(8 \mathrm{ch}, 3-12 \mu \mathrm{m})\end{array}$ & Frame rate & $256 \mathrm{Mpix} / \mathrm{sec}$ at $32 \mathrm{kHz}$ & 2012 & 2016 & Earth \\
\hline UV \& IR CCD arrays & Pixel array: $4 \mathrm{k} \times 4 \mathrm{k}$ & $10 \mathrm{k} \mathrm{x} 10 \mathrm{k}$ & 2011 & 2014 & Earth Astro \\
\hline $\begin{array}{l}\text { UV-VIS spectrometer } \\
\text { Hybrid arrays }\end{array}$ & $\begin{array}{l}\text { Well Depth: } \\
\text { array: } 1 \mathrm{k} \times 1 \mathrm{k}\end{array}$ & $\begin{array}{l}1 \mathrm{M} \text { electrons } 4 \mathrm{kx} \\
4 \mathrm{k}\end{array}$ & 2010 & 2013 & $\begin{array}{l}\text { Earth } \\
\text { Helio }\end{array}$ \\
\hline $\begin{array}{l}\text { UV-VIS-NIR } \\
\text { spectrometer ROIC }\end{array}$ & $\begin{array}{l}\text { Pixel array: } 256 \times 256 \\
\text { Quantization level: } 50 \% \mathrm{QE}\end{array}$ & $\begin{array}{l}1024 \times 2048 \\
>90 \% \text { VIS-NIR }\end{array}$ & 2013 & 2019 & Earth \\
\hline $\begin{array}{l}\text { Backscatter lidar } \\
\text { CCD array }\end{array}$ & Quantum efficiency: & $\begin{array}{l}>70 \% \text { at } 355 \mathrm{~nm} ;>90 \% \\
\text { at } 532 \mathrm{~nm}\end{array}$ & 2012 & 2019 & Earth \\
\hline
\end{tabular}

- Optics: High-throughput with large fields of view, high stability, spectral resolution, and uniformity at many different temperatures.

- Microwave/Radio Transmitters and Receivers: Low-noise amplifier technologies, with reliable low-power high-speed digital- and mixed-signal processing electronics and algorithms.

- Lasers: Reliable, highly stable, Examples from Table 2.2.2.1-1

\begin{tabular}{|c|c|c|c|c|c|}
\hline Technology Metric & State of Art & Need & Start & TRL6 & Mission \\
\hline \multicolumn{6}{|c|}{ 8.1.2.1 Radiation Hardened } \\
\hline $\begin{array}{l}\text { Radiation-hardened } \\
\text { electronics }\end{array}$ & $\begin{array}{l}\text { TID tolerance } \\
0.1-1 \text { Mrad }\end{array}$ & $3 \mathrm{Mrad}$ & 2010 & 2020 & Planet \\
\hline \multicolumn{6}{|l|}{ 8.1.2.2 Low Noise } \\
\hline $\mathrm{ROIC}$ & $\begin{array}{l}\text { Well: }<100 \mathrm{~K} \mathrm{e} \\
\text { Format: } 4 \mathrm{k} \times 4 \mathrm{k} \\
\text { Speed: Low }\end{array}$ & $\begin{array}{l}>2 \mathrm{Me} \\
8 \mathrm{k} \text { X } 8 \mathrm{k} \\
>60 \mathrm{FPS}\end{array}$ & 2013 & 2019 & Earth Astro \\
\hline Low-noise electronics & $\begin{array}{l}\text { Noise level: }<1 \% \\
\text { Temperature }-55 \mathrm{C} \text { to } \\
125 \mathrm{C}\end{array}$ & $\begin{array}{l}<0.01 \% \\
-180 \mathrm{C} \text { to125 } \\
\mathrm{C}\end{array}$ & 2011 & 2020 & $\begin{array}{l}\text { Planet, Astro, Earth, } \\
\text { Helio }\end{array}$ \\
\hline HV power supply & $\begin{array}{l}\text { Voltage out } \\
\text { Eff= 15\%@20 kV, } \\
\text { TID tolerance } 0.1 \mathrm{Mrad}\end{array}$ & $\begin{array}{l}20 \mathrm{kV} \\
>20 \% \\
0.7 \mathrm{Mrad} \\
\end{array}$ & 2013 & 2019 & $\begin{array}{l}\text { Earth } \\
\text { Helio }\end{array}$ \\
\hline \multicolumn{6}{|l|}{ 8.1.2.3 High Speed } \\
\hline Fast electronics & $\begin{array}{l}\text { Timing } 10 \mathrm{~ns} \\
\text { Dead T/event } 300 \mathrm{~ns}\end{array}$ & $\begin{array}{l}\sim 3 \mathrm{~ns} \\
\sim 30 \mathrm{~ns}\end{array}$ & 2012 & 2014 & Helio \\
\hline High-speed: altimetry & Freq: $200 \mathrm{Mz}$ & $2-8 \mathrm{GHz}$ & 2012 & 2020 & Planet \\
\hline
\end{tabular}
efficient, radiation hardened, and long lifetime ( $>5$ years)

- Cryogenic/Thermal Systems: Low power, lightweight, and low vibration 


\section{Push Technologies: 8.1 Science Instruments}

\begin{tabular}{|c|c|}
\hline Push Technology & Description \\
\hline \multicolumn{2}{|c|}{ 8.1 Remote-Sensing Instruments/Sensors } \\
\hline $\begin{array}{l}\text { Quantum Optical } \\
\text { Interferometry }\end{array}$ & $\begin{array}{l}\text { Produce and measure quantum entangled-photons with lasers with the potential to } \\
\text { improve the sensitivity of optical interferometers by multiple orders of magnitude. }\end{array}$ \\
\hline Imaging Lidar & $\begin{array}{l}\text { Imaging Lidar technologies involving fiber lasers and 2D detector arrays will enable } \\
\text { "range imaging" of Earth and planetary surfaces. }\end{array}$ \\
\hline $\begin{array}{l}\text { Atmospheric Trace-Gas } \\
\text { Lidar }\end{array}$ & $\begin{array}{l}\text { Atmospheric trace-gas Lidar technologies for biogenic trace gas measurement and } \\
\text { localization (Earth and Planets) }\end{array}$ \\
\hline $\begin{array}{l}\text { Long Range Laser Induced } \\
\text { Mass Analysis }\end{array}$ & $\begin{array}{l}\text { Long range laser induced mass analysis (LIMA) methods for atmosphere-less bodies } \\
\text { (NEO's, Moon, Mercury, outer planets) }\end{array}$ \\
\hline $\begin{array}{l}\text { Hyper-resolution Visible- } \\
\text { NIR }\end{array}$ & $\begin{array}{l}\text { Hyper-resolution Visible-NIR imaging using TDI detectors and lightweighted optics in } \\
\text { the } 1-1.5 \mathrm{~m} \text { class }(5 \mathrm{~cm} \text {-pixel class) }\end{array}$ \\
\hline K-Band Radar & $\begin{array}{l}\text { Compact K-band imaging and sounding radars (nadir and sidelooking) for planetary } \\
\text { sciences (small antennae, lower power) }\end{array}$ \\
\hline IR Spectrometers & Advanced, multi-detector Fabry Perot IR spectrometers for trace-gas detection \\
\hline Optical Communications & $\begin{array}{l}\text { Mass efficient optical telecommunications systems capable of } 100 \mathrm{Mbps} \text { to } 1 \mathrm{Gbps} \text { from } \\
\text { Mars or Venus orbit (to Earth) or up to } 100 \mathrm{Mbps} \text { from Jupiter or Saturn would increase } \\
\text { bandwidth by a factor of } 10-100 \text { and improve scientific ranging to spacecraft by a factor } \\
\text { of } 10-50 \text { over RF methods. }\end{array}$ \\
\hline Lidar Fiber Transmitters & $\begin{array}{l}\text { Advanced fiber-based laser transmitters with } 0.01 \text { to } 20 \mathrm{~mJ} \text { pulse energy in the Green to } \\
\text { NIR for lidars }\end{array}$ \\
\hline 3-D Imaging Flash Lidar & $\begin{array}{l}\text { 3-D Imaging Flash Lidar for Safe landing on planetary bodies by enabling Hazard } \\
\text { Detection and Avoidance. 3-D Imaging Flash Lidar has also been identified as the } \\
\text { primary sensor for Automatic Rendezvous and Docking. }\end{array}$ \\
\hline Radar 3-D Imaging & $\begin{array}{l}\text { Shallow, radar 3D imaging via a sounding-imaging-SAR would allow the lunar regolith } \\
\text { to be mapped in 3D at spatial scales of } 10-20 \mathrm{~m} \text { and vertically to } 3-5 \mathrm{~m} \text {; the same could be } \\
\text { done for Europa or NEO's }\end{array}$ \\
\hline Hyper-Resolution SAR & $\begin{array}{l}\text { Hyper-resolution SAR enabled by wideband electronically steered array based } \\
\text { technologies and advanced T/R switches and microwave power modules could enable } \\
\text { sub-meter RADAR imaging of cloud-enshrouded planets such as Titan and Venus at } \\
\text { scales of } 50 \mathrm{~cm} \text { to } 1 \mathrm{~m} \text { and have the equivalent impact as the optical high resolution } \\
\text { maging at Mars and the Moon (HiRISE and LROC) }\end{array}$ \\
\hline Extended-Life IR Sensors & $\begin{array}{l}\text { The first essential ingredient for success for a human mission to a NEO is to complete } \\
\text { the NEO survey to identify the most interesting human-accessible targets. A space- } \\
\text { based IR survey telescope in a heliocentric orbit } 0.65 \text { to } 0.72 \text { Astronomical Units (AU) } \\
\text { from the Sun will enable mapping of the remaining NEOs not visible from Earth-based } \\
\text { observatories and identification of the orbital dynamic characteristics. }\end{array}$ \\
\hline $\begin{array}{l}\text { Soil Moisture using L-band } \\
\text { GPS }\end{array}$ & $\begin{array}{l}\text { Use the earth-surface "bounced" L-band GPS signal to measure changes in soil moisture } \\
\text { with time to improve crop yields and climate models that utilize soil moisture. }\end{array}$ \\
\hline $\begin{array}{l}\text { Ocean wind speed } \\
\text { measurement }\end{array}$ & $\begin{array}{l}\text { Deploy small GPS bistatic receivers on commercial cargo aircraft to utilize ocean- } \\
\text { reflected ("bounced") GPS signals for ocean wind speed measurement. Since GPS is } \\
\text { available globally, high-resolution wind speed measurements can be taken over large } \\
\text { portions of the ocean to study detailed weather patterns and storm development. }\end{array}$ \\
\hline
\end{tabular}




\subsection{Remote Sensing Instruments/Sensors}

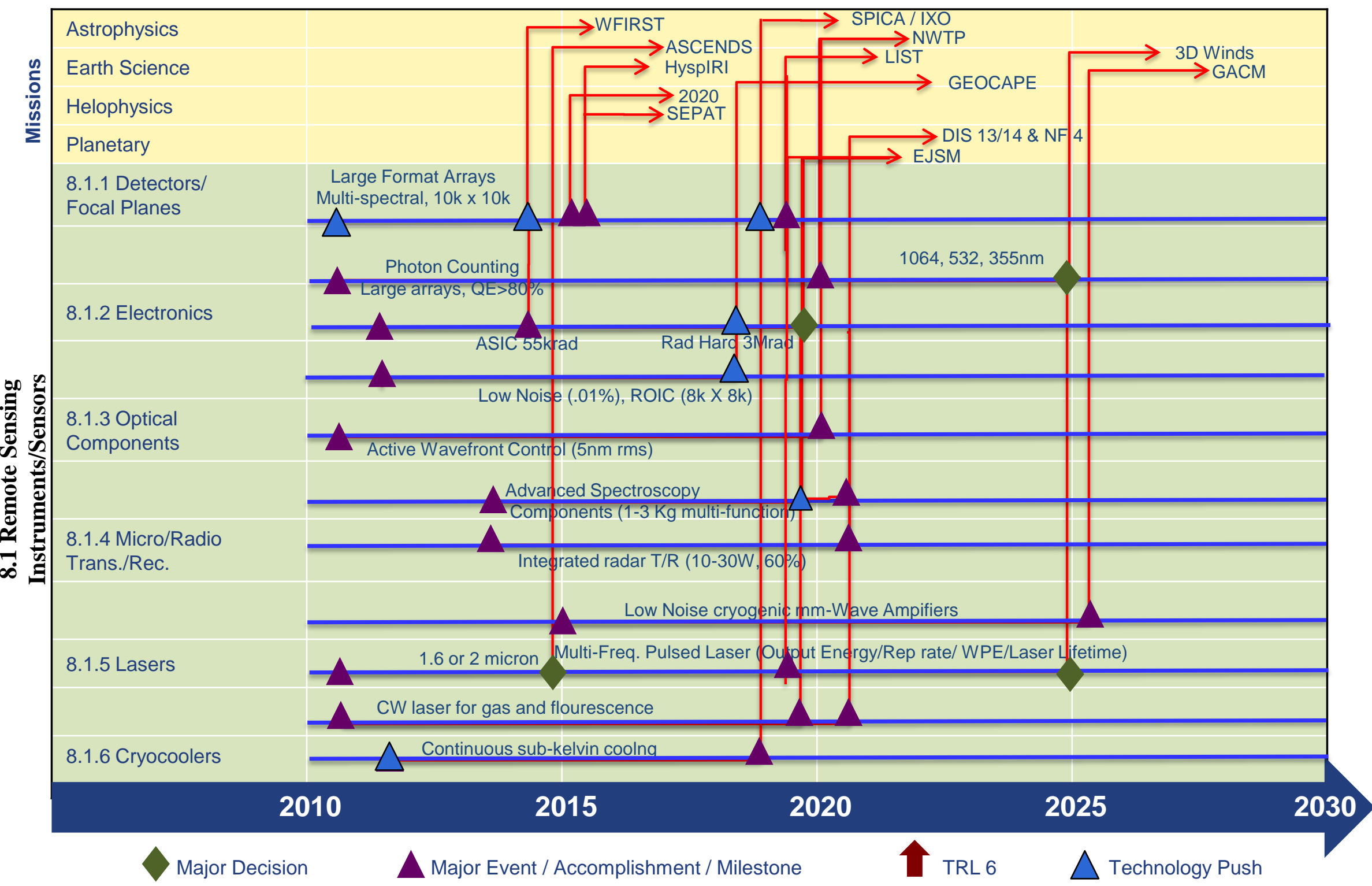




\section{Technology Area 8.2 Observatory}

Major challenges include:

$\mathrm{X}$-ray Grazing Incidence Mirror Systems

UV-Vis-IR Normal Incidence Mirror Systems

Large Ultra-stable Structures

Large Deployable/Assembled Structures

Control of Large Structures

Distributed Aperture / Formation flying

Technologies support 3 applications:

$\mathrm{X}$-ray astronomy,

UVOIR astronomy, and

Radio / microwave antenna.

Most important metric for all observatories is cost per square meter of aperture.

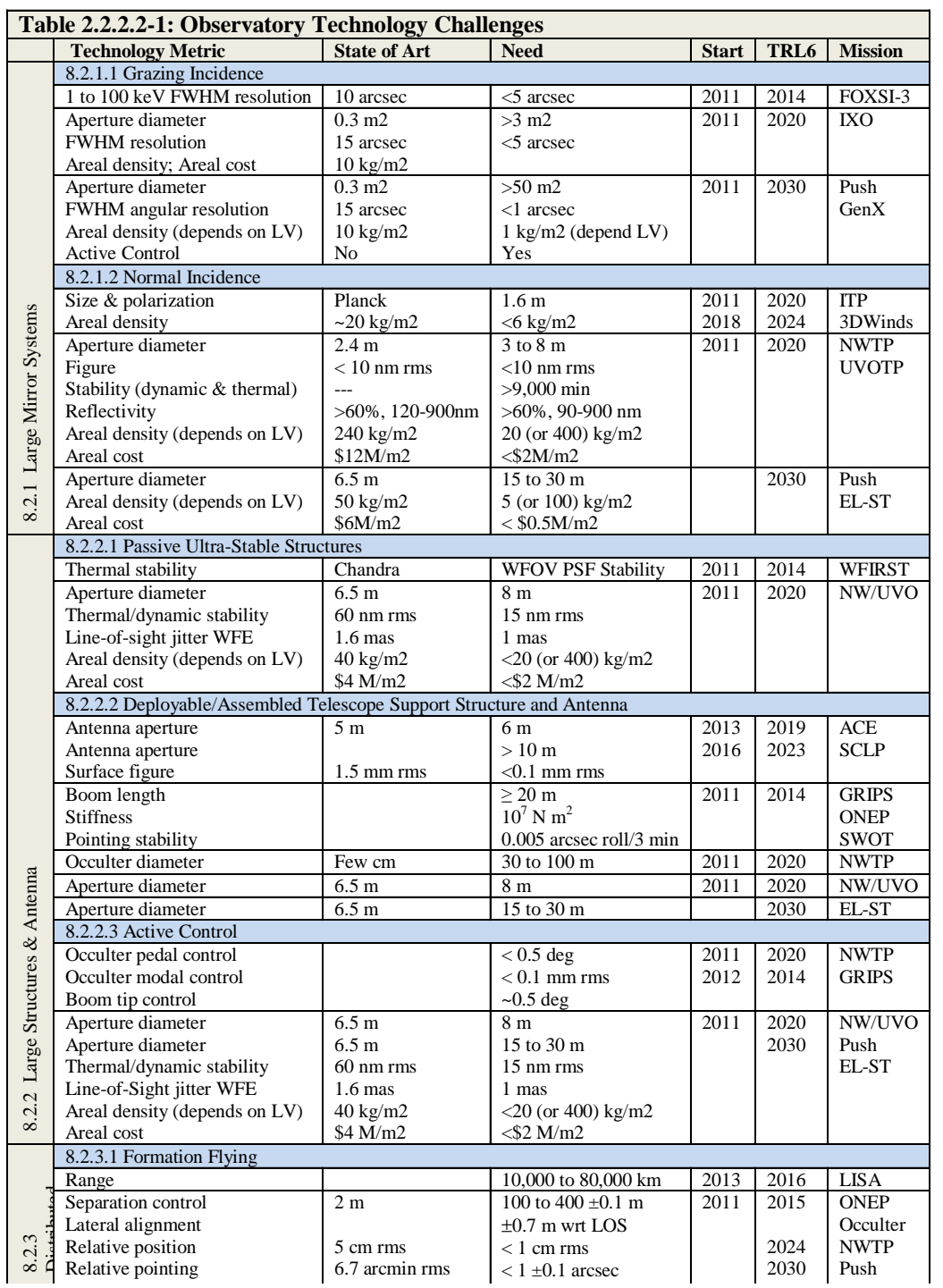


\begin{tabular}{|l}
\hline Table 2.2.2.2-1: Observatory Technology Challenges \\
\hline
\end{tabular}

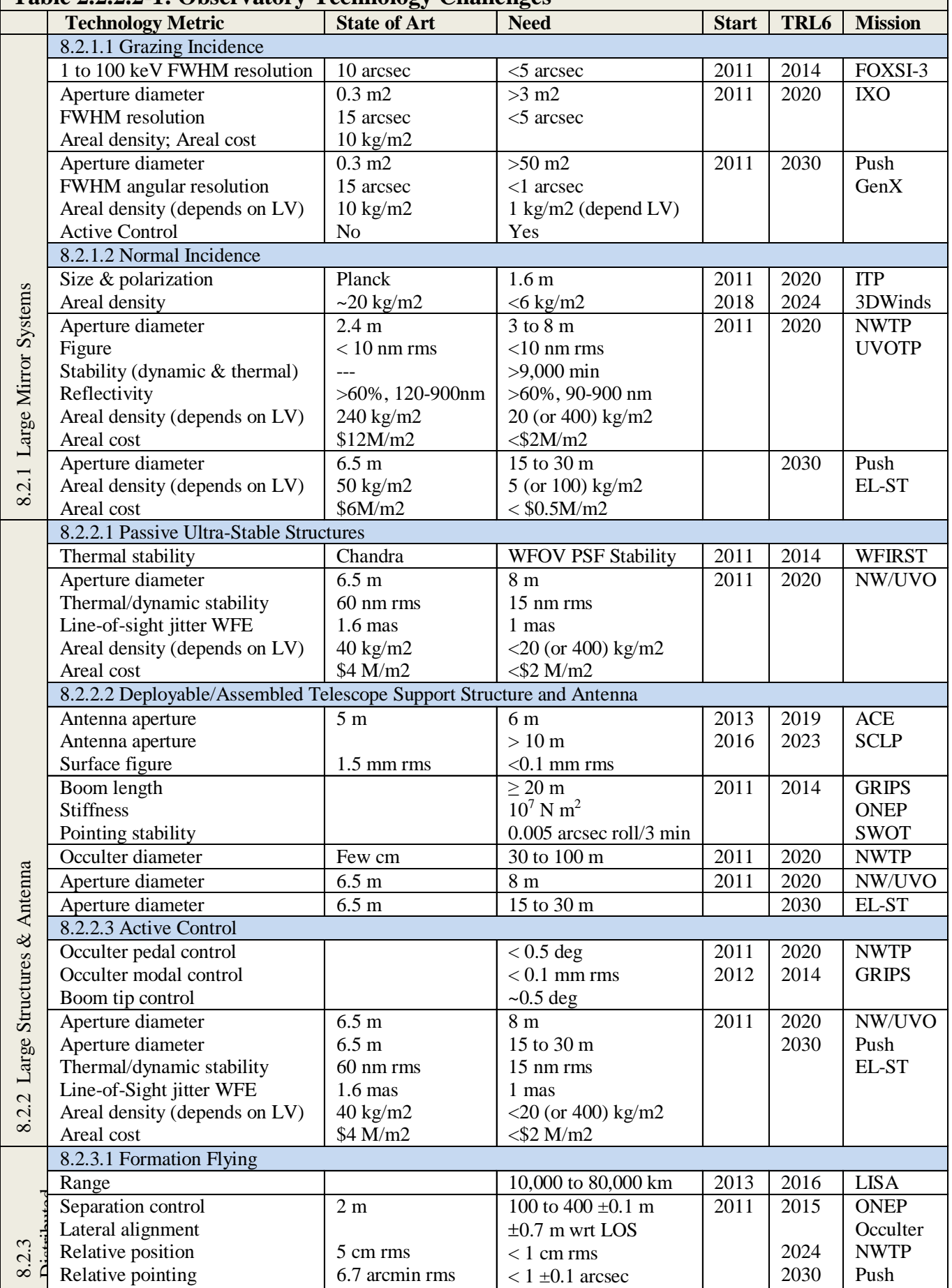




\section{Observatory Budget Recommendations}

\$400M over 10-yrs to Industry/Academia for X-Ray mirrors, large UV mirrors, large structures, and formation flying:

$\begin{array}{llll}\text { Program } & 10 \text { year } & 2012 & 2021 \\ \text { IXO } & \$ 150 \mathrm{M} & \$ 3 \mathrm{M} / \mathrm{yr} & \$ 20 \mathrm{M} / \mathrm{yr} \\ \text { New World } & \$ 100 \mathrm{M} & \$ 2 \mathrm{M} / \mathrm{yr} & \$ 15 \mathrm{M} / \mathrm{yr} \\ \text { UVO } & \$ 20 \mathrm{M} & \$ 1 \mathrm{M} / \mathrm{yr} & \$ 5 \mathrm{M} / \mathrm{yr} \\ \text { General } & \$ 100 \mathrm{M} & \$ 10 \mathrm{M} / \mathrm{yr} & \$ 10 \mathrm{M} / \mathrm{yr} \\ \text { Earth/Helio } & \$ 30 \mathrm{M} & \$ 1 \mathrm{M} / \mathrm{yr} & \$ 5 \mathrm{M} / \mathrm{yr} \\ \text { TOTAL } & \$ 400 \mathrm{M} & \$ 17 \mathrm{M} / \mathrm{yr} & \$ 55 \mathrm{M} / \mathrm{yr}\end{array}$

Plus another $\$ 200 \mathrm{M}$ over 10-years for Internal NASA funding $75 \mathrm{FTE} / \mathrm{yr} \& \$ 5 \mathrm{M} / \mathrm{yr}$ ODC 


\begin{tabular}{|c|c|c|c|c|c|c|c|c|c|c|c|}
\hline \multirow[t]{2}{*}{ ano } & \multirow[t]{2}{*}{ Technology Metric } & \multirow[t]{2}{*}{ State of Art } & \multirow[t]{2}{*}{ Need } & \multirow[t]{2}{*}{ TRL } & \multirow[t]{2}{*}{ TRL6 } & \multirow[t]{2}{*}{ Mission } & \multicolumn{3}{|c|}{ 10-yr External } & \multicolumn{2}{|c|}{ NASA Internal } \\
\hline & & & & & & & Total & FY12 & FY21 & FTE/yr & ODC/yr \\
\hline & \multicolumn{6}{|l|}{ 8.2 Observatory Technology } & $\$ 400 M$ & $\$ 19 M$ & $\$ 48 M$ & $75 / y r$ & $\$ 5 \mathrm{M} / \mathrm{yr}$ \\
\hline \multirow{8}{*}{ 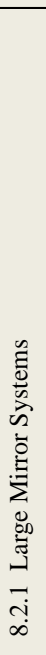 } & \multicolumn{6}{|l|}{ 8.2.1.1 Grazing Incidence } & $\$ 170 \mathrm{M}$ & $\$ 6 \mathrm{M}$ & $\$ 22 \mathrm{M}$ & 30 & $\$ 2 \mathrm{M}$ \\
\hline & 1 to $100 \mathrm{keV}$ FWHM resolution & $10 \operatorname{arcsec}$ & $<5 \operatorname{arcsec}$ & 5 & 2014 & FOXSI-3 & 5 & 2 & - & 6 & .5 \\
\hline & $\begin{array}{l}\text { Aperture diameter } \\
\text { FWHM resolution } \\
\text { Areal density; Areal cost }\end{array}$ & $\begin{array}{l}0.3 \mathrm{~m} 2 \\
15 \mathrm{arcsec} \\
10 \mathrm{~kg} / \mathrm{m} 2\end{array}$ & $\begin{array}{l}>3 \mathrm{~m} 2 \\
<5 \operatorname{arcsec}\end{array}$ & 3 & 2020 & IXO & 150 & 3 & 20 & 22 & 1.5 \\
\hline & $\begin{array}{l}\text { Aperture diameter } \\
\text { FWHM angular resolution } \\
\text { Areal density (depends LV) } \\
\text { Active Control }\end{array}$ & $\begin{array}{l}0.3 \mathrm{~m} 2 \\
15 \operatorname{arcsec} \\
10 \mathrm{~kg} / \mathrm{m} 2 \\
\text { No }\end{array}$ & $\begin{array}{l}>50 \mathrm{~m} 2 \\
<1 \operatorname{arcsec} \\
1 \mathrm{~kg} / \mathrm{m} 2 \text { (depend LV) } \\
\text { Yes }\end{array}$ & 2 & 2030 & $\begin{array}{l}\text { Push } \\
\text { GenX }\end{array}$ & 15 & 1 & 2 & 2 & - \\
\hline & \multicolumn{6}{|l|}{ 8.2.1.2 Normal Incidence } & $\$ 80 \mathrm{M}$ & $\$ 3 \mathrm{M}$ & $\$ 8 \mathrm{M}$ & 15 & $\$ 1 \mathrm{M}$ \\
\hline & $\begin{array}{l}\text { Size \& polarization } \\
\text { Areal density }\end{array}$ & $\begin{array}{l}\text { Planck } \\
\sim 20 \mathrm{~kg} / \mathrm{m} 2\end{array}$ & $\begin{array}{l}1.6 \mathrm{~m} \\
<6 \mathrm{~kg} / \mathrm{m} 2\end{array}$ & $\begin{array}{l}5 \\
5\end{array}$ & $\begin{array}{l}2020 \\
2024\end{array}$ & $\begin{array}{c}\text { ITP } \\
\text { 3DWinds }\end{array}$ & 5 & 1 & - & 3 & - \\
\hline & $\begin{array}{l}\text { Aperture diameter } \\
\text { Figure } \\
\text { Stability (dynamic \& thermal) } \\
\text { Reflectivity } \\
\text { Areal density (depends LV) } \\
\text { Areal cost }\end{array}$ & $\begin{array}{l}2.4 \mathrm{~m} \\
<10 \mathrm{~nm} \mathrm{rms} \\
--- \\
>60 \%, 120-900 \mathrm{~nm} \\
240 \mathrm{~kg} / \mathrm{m} 2 \\
\$ 12 \mathrm{M} / \mathrm{m} 2\end{array}$ & $\begin{array}{l}3 \text { to } 8 \mathrm{~m} \\
<10 \mathrm{~nm} \mathrm{rms} \\
>9,000 \mathrm{~min} \\
>60 \%, 90-900 \mathrm{~nm} \\
20(\text { or } 400) \mathrm{kg} / \mathrm{m} 2 \\
<\$ 2 \mathrm{M} / \mathrm{m} 2\end{array}$ & 4 & 2020 & $\begin{array}{l}\text { NWTP } \\
\text { UVOTP }\end{array}$ & 75 & 2 & 9 & 10 & 1 \\
\hline & $\begin{array}{l}\text { Aperture diameter } \\
\text { Areal density (depends LV) } \\
\text { Areal cost }\end{array}$ & $\begin{array}{l}6.5 \mathrm{~m} \\
50 \mathrm{~kg} / \mathrm{m} 2 \\
\$ 6 \mathrm{M} / \mathrm{m} 2\end{array}$ & $\begin{array}{l}15 \text { to } 30 \mathrm{~m} \\
5(\text { or } 100) \mathrm{kg} / \mathrm{m} 2 \\
<\$ 0.5 \mathrm{M} / \mathrm{m} 2\end{array}$ & 2 & 2030 & $\begin{array}{l}\text { Push } \\
\text { EL-ST }\end{array}$ & TBD & TBD & TBD & 2 & - \\
\hline \multirow{12}{*}{ 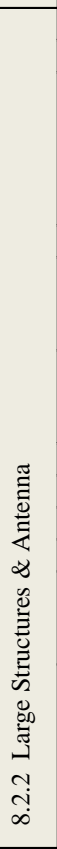 } & \multicolumn{6}{|c|}{\begin{tabular}{|l|}
8.2 .2 .1 Passive Ultra-Stable Structures \\
\end{tabular}} & $\$ 20 \mathrm{M}$ & $\$ 3 \mathrm{M}$ & $\$ 2 \mathrm{M}$ & 4 & $\$ 0.3 \mathrm{M}$ \\
\hline & Thermal stability & Chandra & WFOV PSF Stability & 5 & 2014 & WFIRST & 5 & 2 & - & 2 & - \\
\hline & $\begin{array}{l}\text { Aperture diameter } \\
\text { Thermal/dynamic stability } \\
\text { Line-of-sight jitter WFE } \\
\text { Areal density (depends LV) } \\
\text { Areal cost }\end{array}$ & $\begin{array}{l}6.5 \mathrm{~m} \\
60 \mathrm{~nm} \mathrm{rms} \\
1.6 \mathrm{mas} \\
40 \mathrm{~kg} / \mathrm{m} 2 \\
\$ 4 \mathrm{M} / \mathrm{m} 2 \\
\end{array}$ & $\begin{array}{l}8 \mathrm{~m} \\
15 \mathrm{~nm} \mathrm{rms} \\
1 \mathrm{mas} \\
<20(\text { or } 400) \mathrm{kg} / \mathrm{m} 2 \\
<\$ 2 \mathrm{M} / \mathrm{m} 2\end{array}$ & 3 & 2020 & NW/UVO & 15 & 1 & 2 & 2 & .3 \\
\hline & \multicolumn{6}{|c|}{ 8.2.2.2 Deployable/Assembled Telescope Support Structure and Antenna } & $\$ 50 \mathrm{M}$ & $\$ 4 \mathrm{M}$ & $\$ 6 \mathrm{M}$ & 10 & $\$ 0.7 \mathrm{M}$ \\
\hline & $\begin{array}{l}\text { Antenna aperture } \\
\text { Antenna aperture } \\
\text { Surface figure }\end{array}$ & $\begin{array}{l}5 \mathrm{~m} \\
1.5 \mathrm{~mm} \mathrm{rms}\end{array}$ & $\begin{array}{l}6 \mathrm{~m} \\
>10 \mathrm{~m} \\
<0.1 \mathrm{~mm} \mathrm{rms}\end{array}$ & $\begin{array}{l}5 \\
3\end{array}$ & $\begin{array}{l}2019 \\
2023\end{array}$ & $\begin{array}{l}\text { ACE } \\
\text { SCLP }\end{array}$ & 5 & 1 & - & 1 & - \\
\hline & $\begin{array}{l}\text { Boom length } \\
\text { Stiffness } \\
\text { Pointing stability }\end{array}$ & & $\begin{array}{l}\geq 20 \mathrm{~m} \\
10^{7} \mathrm{~N} \mathrm{~m}^{2} \\
0.005 \mathrm{arcsec} \mathrm{roll} / 3 \mathrm{~min}\end{array}$ & 5 & 2014 & $\begin{array}{l}\text { GRIPS } \\
\text { ONEP } \\
\text { SWOT }\end{array}$ & 5 & 2 & - & 3 & .3 \\
\hline & Occulter diameter & Few $\mathrm{cm}$ & 30 to $100 \mathrm{~m}$ & 2 & 2020 & NWTP & 20 & 1 & 3 & 3 & .3 \\
\hline & Aperture diameter & $6.5 \mathrm{~m}$ & $8 \mathrm{~m}$ & 4 & 2020 & NW/UVO & 20 & 1 & 3 & 2 & .1 \\
\hline & Aperture diameter & $6.5 \mathrm{~m}$ & 15 to $30 \mathrm{~m}$ & 2 & 2030 & EL-ST & TBD & TBD & TBD & 1 & - \\
\hline & \multicolumn{6}{|l|}{ 8.2.2.3 Active Control } & $\$ 30 \mathrm{M}$ & $\$ 2 \mathrm{M}$ & $\$ 4 \mathrm{M}$ & 6 & $\$ 0.4 \mathrm{M}$ \\
\hline & $\begin{array}{l}\text { Occulter pedal control } \\
\text { Occulter modal control } \\
\text { Boom tip control }\end{array}$ & & $\begin{array}{l}<0.5 \mathrm{deg} \\
<0.1 \mathrm{~mm} \mathrm{rms} \\
\sim 0.5 \mathrm{deg}\end{array}$ & $\begin{array}{l}3 \\
5\end{array}$ & $\begin{array}{l}2020 \\
2014\end{array}$ & $\begin{array}{l}\text { NWTP } \\
\text { GRIPS }\end{array}$ & 15 & 1 & 2 & 3 & .2 \\
\hline & $\begin{array}{l}\text { Aperture diameter } \\
\text { Aperture diameter } \\
\text { Thermal/dynamic stability } \\
\text { Line-of-Sight jitter WFE } \\
\text { Areal density (depends LV) } \\
\text { Areal cost }\end{array}$ & $\begin{array}{l}6.5 \mathrm{~m} \\
6.5 \mathrm{~m} \\
60 \mathrm{~nm} \mathrm{rms} \\
1.6 \mathrm{mas} \\
40 \mathrm{~kg} / \mathrm{m} 2 \\
\$ 4 \mathrm{M} / \mathrm{m} 2 \\
\end{array}$ & $\begin{array}{l}8 \mathrm{~m} \\
15 \text { to } 30 \mathrm{~m} \\
15 \mathrm{~nm} \mathrm{rms} \\
1 \mathrm{mas} \\
<20(\text { or } 400) \mathrm{kg} / \mathrm{m} 2 \\
<\$ 2 \mathrm{M} / \mathrm{m} 2\end{array}$ & $\begin{array}{l}3 \\
2\end{array}$ & $\begin{array}{l}2020 \\
2030\end{array}$ & $\begin{array}{l}\text { NW/UVO } \\
\text { Push } \\
\text { EL-ST }\end{array}$ & 15 & 1 & 2 & 3 & .2 \\
\hline \multirow[b]{3}{*}{ 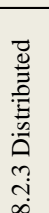 } & \multicolumn{6}{|l|}{ 8.2.3.1 Formation Flying } & $\$ 50 \mathrm{M}$ & $\$ 1 \mathrm{M}$ & $\$ 7 \mathrm{M}$ & 10 & $\$ 0.6 \mathrm{M}$ \\
\hline & Range & & 10,000 to $80,000 \mathrm{~km}$ & 5 & 2016 & LISA & TBD & TBD & TBD & 1 & - \\
\hline & $\begin{array}{l}\text { Separation control } \\
\text { Lateral alignment } \\
\text { Relative position } \\
\text { Relative pointing }\end{array}$ & $\begin{array}{l}2 \mathrm{~m} \\
5 \mathrm{~cm} \mathrm{rms} \\
6.7 \mathrm{arcmin} \mathrm{rms}\end{array}$ & $\begin{array}{l}100 \text { to } 400 \pm 0.1 \mathrm{~m} \\
\pm 0.7 \mathrm{~m} \mathrm{wrt} \mathrm{LOS} \\
<1 \mathrm{~cm} \mathrm{rms} \\
<1 \pm 0.1 \mathrm{arcsec}\end{array}$ & $\begin{array}{l}5 \\
3 \\
2\end{array}$ & $\begin{array}{l}2015 \\
2024 \\
2030\end{array}$ & $\begin{array}{l}\text { ONEP } \\
\text { Occulter } \\
\text { NWTP } \\
\text { Push }\end{array}$ & 50 & 1 & 7 & 9 & .6 \\
\hline
\end{tabular}




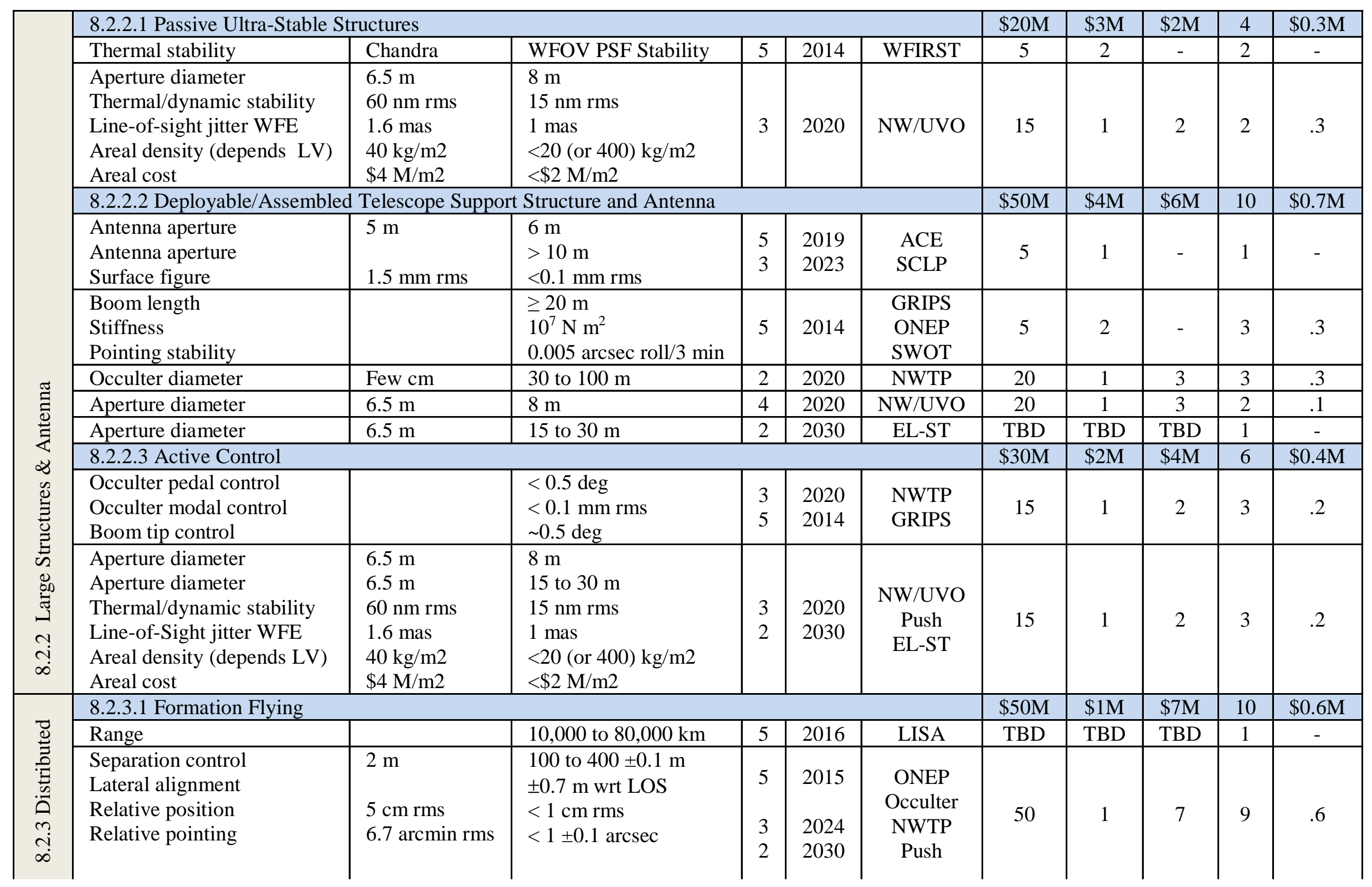




\section{Push Technologies: 8.2 Observatories}

\begin{tabular}{|c|c|}
\hline Technology & Description \\
\hline \multicolumn{2}{|l|}{8.2 Observatories } \\
\hline $\begin{array}{l}\text { Synthetic Aperture Imaging } \\
\text { Lidar (SAIL) }\end{array}$ & $\begin{array}{l}\text { Synthetic Aperture Imaging Lidar (SAIL) for hyper-resolution imaging and 3D ranging } \\
\text { (range imaging). SAIL methods could map dynamics of planetary surfaces on Mars } \\
\text { (polar caps), Titan (moving landscapes), and even on Europa much more efficiently than } \\
\text { current single beam or multi-beam approaches. SAIL may be a method worth pursuing } \\
\text { for ICESat-3 in the } 2020^{\prime} \text { s to rapidly build up 3D geodetic maps of the ice covered } \\
\text { surfaces of Earth }\end{array}$ \\
\hline $\begin{array}{l}\text { Super High-Resolution } \\
\text { Imaging of High-Energy } \\
\text { Photons }\end{array}$ & $\begin{array}{l}\text { The technology need is to build a large area (much larger than current optics) high } \\
\text { energy optic and then have it fly it formation with the imaging spacecraft }\end{array}$ \\
\hline Radar Arrays & Wideband active electronically steered array radar with lightweighted antennae \\
\hline Precision Interferometry & $\begin{array}{l}\text { Requires CW single-frequency and frequency-stabilized lasers for space (GSFC } \\
\text { applications so far are pulsed). Digital techniques including coded modulation for time- } \\
\text { of-flight resolvable interference, and flexible in-flight changes. Time-Domain } \\
\text { Interferometry (LISA's equal-path-length synthesis techniques). }\end{array}$ \\
\hline $\begin{array}{l}\text { Hyper-Resolution Visible- } \\
\text { NIR }\end{array}$ & $\begin{array}{l}\text { Hyper-resolution Visible-NIR imaging using lightweighted optics in the } 1-1.5 \mathrm{~m} \text { class ( } 5 \\
\mathrm{~cm} / \text { pixel class) }\end{array}$ \\
\hline K-Band Radar & $\begin{array}{l}\text { Compact K-band imaging and sounding radars (nadir and sidelooking) for planetary } \\
\text { sciences (small antennae) }\end{array}$ \\
\hline $\begin{array}{l}\text { Conductive Carbon } \\
\text { Nanotubes }\end{array}$ & $\begin{array}{l}\text { Spectacular new material for the fabrication of lightweight antennas could be enabled by } \\
\text { the unbelievable conductivity of individual carbon nanotubes. }\end{array}$ \\
\hline $\begin{array}{l}\text { Deployable Large Aperture } \\
\text { Telescopes }\end{array}$ & $\begin{array}{l}\text { Ultra low mass/volume large deployable large aperture telescopes ( }>2 \text { meter) } \\
\text { for direct detection LIDAR. Concepts include inflatable fresnel, deployable } \\
\text { reflector and petal-based techniques. }\end{array}$ \\
\hline $\begin{array}{l}\text { High stability optical } \\
\text { platforms }\end{array}$ & $\begin{array}{l}\text { Includes optical benches, telescopes, etc, requiring passive thermal isolation for } \\
\text { temperature stability. Hydroxide or silicate bonding for precision alignment capability } \\
\text { and dimensional stability. Precision materials such as Silicon Carbide and single crystal } \\
\text { silicon, Zerodur }\end{array}$ \\
\hline
\end{tabular}




\subsection{Observatories Roadmap}

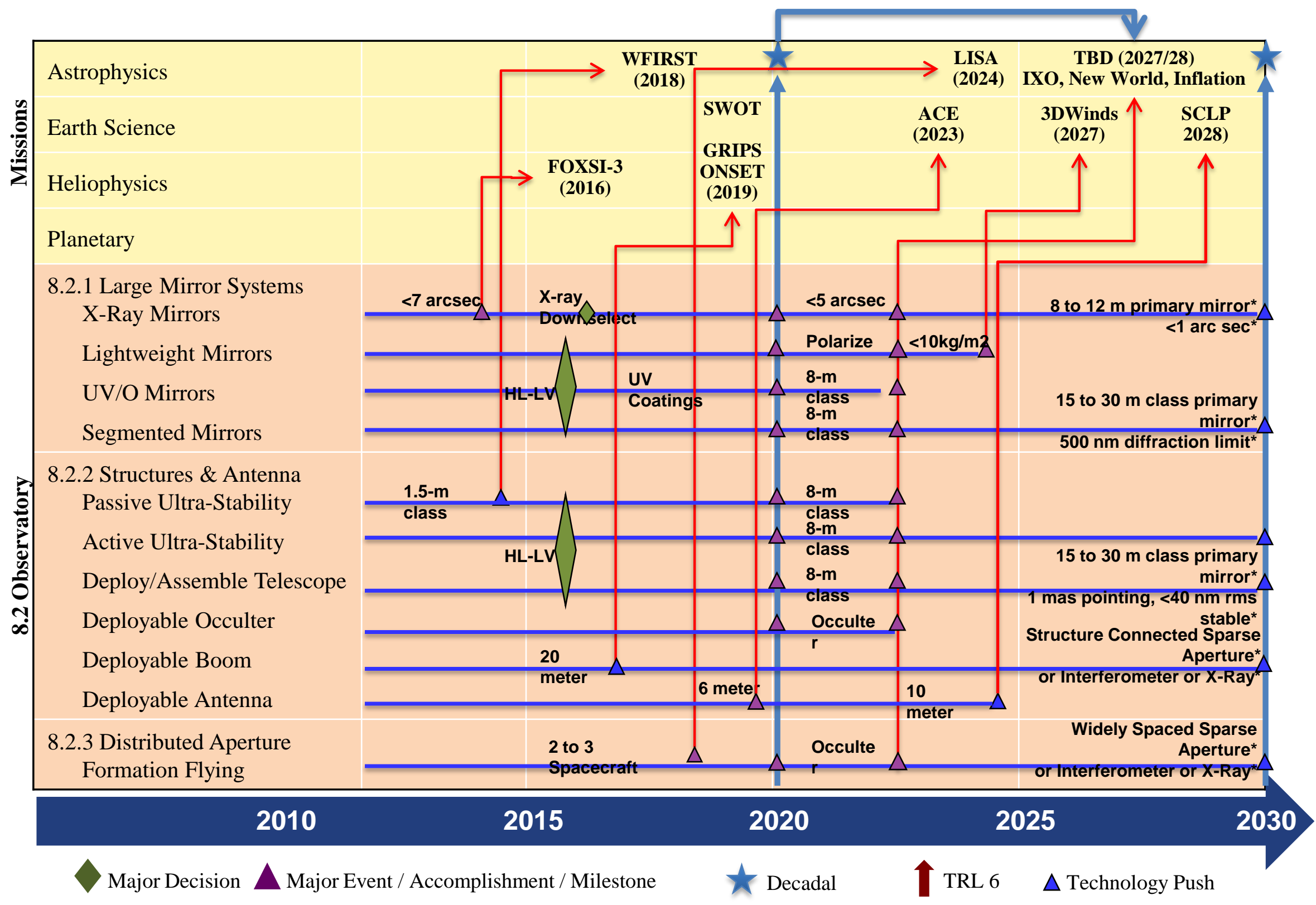




\section{Observatory Technology Needs}

Regardless of whether the incumbent is $0.5 \mathrm{~m}$ or $5 \mathrm{~m}$, the driving need is larger aperture with similar or better performance.

The technologies for achieving performance are

the ability to manufacture and test large-mirror systems;

the structure's ability to hold the mirror in a stable, strain-free state under the influence of anticipated dynamic and thermal stimuli; and, for extra-large apertures, a method to create the aperture via deployment, assembly, or formation flying - where formation-flying technology is simply an actively controlled virtual structure.

One non-telescope application is the manufacture, deployment, in-plane and formation-flying control of an external-occulting starshade to block starlight for exo-planet observation. 


\section{Other Technology Assessment Observatory Needs}

The ability to produce large aperture observatories depends upon advances in other technology assessment areas:

- volume and mass capacities of launch vehicles;

- validated performance models that integrate optical, mechanical, dynamic, and thermal models for telescopes, structures, instruments, and spacecraft to enable the design and manufacture of observatories whose performance requirements are too precise to be tested on the ground;

- new materials and design concepts to enable ultra-stable very large space structures;

- terabit communication; and

- autonomous rendezvous and docking for on-orbit assembly of very large structures. 


\section{Technology Area 8.3 Sensor Systems}

Major challenges include:

Particle and Plasma Sensors

- Energetic Particle Detectors ( $>30 \mathrm{keV}-\mathrm{N} \mathrm{MeV}$ )

- Plasma Detectors $(<1 \mathrm{eV}-30 \mathrm{keV})$

- Magnetometers (DC \& AC)

Fields and Waves Sensors

- EM Field Sensors (DC \& AC)

- Gravity-Wave Sensors

\section{In-Situ Sensors}

- Sample Handling, Preparation, and Containment

- Chemical and Mineral Assessment (Beyond APXS)

- Organic Assessment (Beyond INMS)

- Biological Detection \& Characterization

- Planetary Protection (PP)

Techniques for acquiring, processing, transferring, delivering, and storing subsurface samples are critical and represent a huge gap between needed and available in-situ sensor technologies

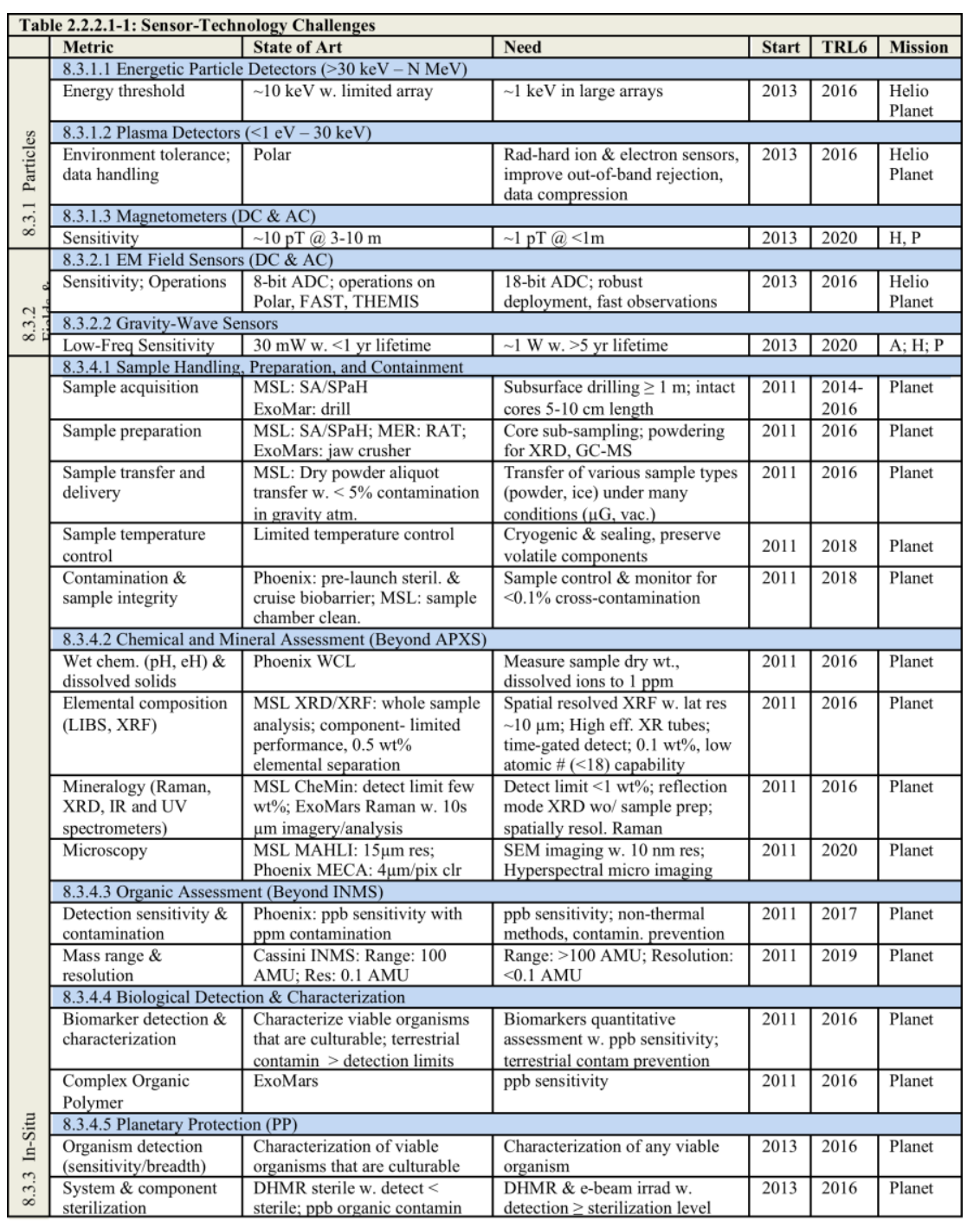




\section{Push Technologies: 8.3 Sensor Systems}

\begin{tabular}{|l|l|}
\hline \multicolumn{1}{|c|}{ Technology } & \multicolumn{1}{c|}{ Description } \\
\hline 8.3 In-Situ Instruments/Sensors \\
\hline Atomic Magnetometers & $\begin{array}{l}\text { This technology has the potential to greatly reduce the resources required to execute } \\
\text { vector magnetic field measurements. }\end{array}$ \\
\hline Neutron Spectroscopy & $\begin{array}{l}\text { In situ dynamic neutron spectroscopy with active sources and collimated detectors } \\
\text { (beyond MSL's DAN) }\end{array}$ \\
\hline $\begin{array}{l}\text { Scanning Electron } \\
\text { Microscope }\end{array}$ & In-situ scanning electron microscope imaging at 1 um and smaller for planetary surfaces \\
\hline X-Ray Imaging & In-situ X-ray imaging for definitive mineralogy without sample preparation \\
\hline $\begin{array}{l}\text { Human Tissue Equivalent } \\
\text { Proportional Radiation } \\
\text { Counter (TEPC) }\end{array}$ & $\begin{array}{l}\text { Current SOA is a space station devices operating in near-atmospheric condition that } \\
\text { measure dosages on crew. Robust sensors capable of operating for long periods in } \\
\text { environment of space are needed to measure the radiation at the destination as well as } \\
\text { during the journey. Previous TEPCs on Mars missions have mostly failed en-route. } \\
\text { Until we get better data on interplanetary environment, the JSC human health group } \\
\text { wants to limit human trips to 150 days or less. }\end{array}$ \\
\hline $\begin{array}{l}\text { Tricorder Health } \\
\text { Monitoring System }\end{array}$ & $\begin{array}{l}\text { As a related topic to humans in space, a monitoring system that will provide a reading of } \\
\text { astronauts' health. }\end{array}$ \\
\hline
\end{tabular}




\subsection{Sensor Systems}

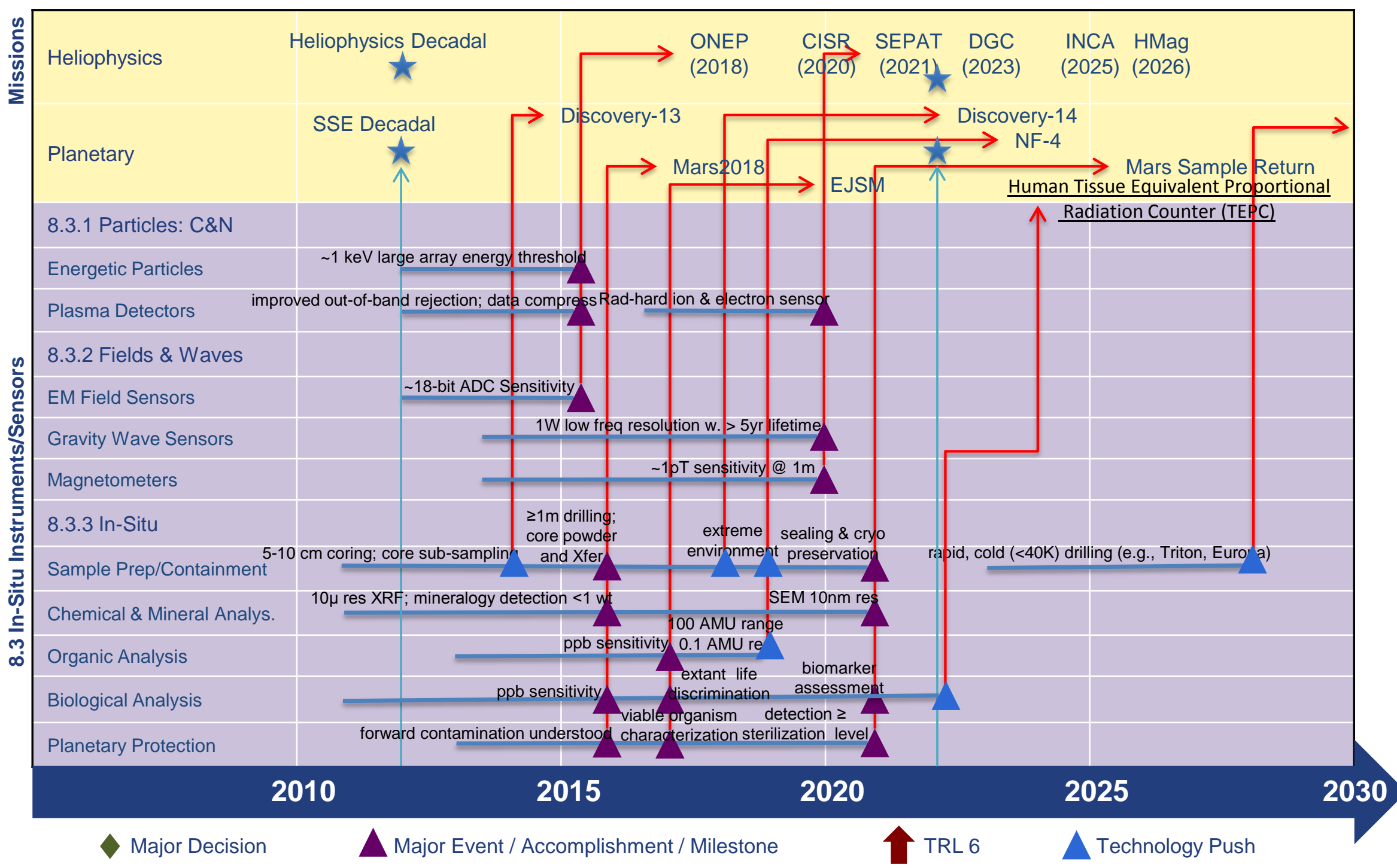




\section{Remote Sensing Instruments/Sensors Public Inputs}

Detectors and Focal Planes:

CZT detectors for x-ray or gamma-ray; next-generation solar-blind photocathodes; TES detectors; CMB detectors; BGO scintillators; UV photon counting detectors; NIR photon counting detectors; detector with small pixels than specified; detector arrays of size larger than specified; detectors with lower noise than specified.

Electronics:

5 for ASIC; 3 on miniaturizing; \& 2 each regarding multiplexers, lownoise amplifiers \& gravity wave phase sensor electronics.

Optical Components:

2 for WFSC to correct phase, intensity, amplitude \& polarization; 4 for components ranging from $\mathrm{x}$-ray \& UV diffraction gratings to narrow band spectral filters to electronically steerable laser beam; 3 microwave polarization feed horns and planar antenna.

3 Radio/Microwave; 1 Lasers and 1 Cryogenic/Thermal. 


\section{Public Inputs}

\section{Observatory:}

8m UVOIR and 4m UVOIR telescopes, 100 meter microwave antenna, high reflectance UV coatings, $\mathrm{x}$-ray and gamma ray imaging optics on 20 meter booms, athermal telescope structures, 400 sq meter microwave phased array antenna structure, 300 meter booms for atom interferometers and distributed aperture systems.

\section{In-Situ Instruments/Sensors:}

1 regarding neutral ion detection, 4 regarding atomic clocks, 5 regarding gravity wave detection, 1 for quantum communication, 1 for mineral assessment and 1 other. 


\section{Acronyms}

ACE - Aerosol/Cloud/Ecosystems

ADC - Analog to Digital Converter

AMU - Atomic Mass Unit

AO - Autonomous Operation

APD - Avalanche Diodes

APIO - Advanced Planning and Integration Office

AR\&D - Applied Research and Development

ASCENDS - Active Sensing of CO2 Emissions over Nights, Days, and Seasons

ASIC - Application Specific Integrated Circuit

ATLAST - Advanced Technology Large Aperture Space Telescope APXS - Alpha Particle X-Ray Spectrometer

AU - Astronomical Units

BEP — Beamed Energy Propulsion

CCD - Charged Coupled Device

CheMin - Chemical Mineral Instrument

CISR - Climate Impacts of Space Radiation

$\mathrm{COM}$ - Communications

$\mathrm{CW}$ - Continuous Wave

DIAL - Differential Absorption Lidar

DGC — Dynamic Geospace Coupling

DHMR — Dry Heat Microbial Reduction

EDL - Entry, Descent and Landing

EJSM — Europa-Jupiter System Mission

ELST — Extremely Large Space Telescopes

EM - Electromagnetic

EMS - Environmental Monitoring and Safety

FAST - Fast Auroral SnapshoT

FOV - Field of View

FOXSI - Focusing Optics X-ray Solar Imager
FPA - Focal Plane Array

FWHM-Full Width Half Maximum

GACM - Global Atmospheric Composition Mission

GC-MS - Gas Chromatography-Mass Spectroscopy

GenX - Generation-X Vision

GEO - Geosynchronous Orbit

GEO-CAPE - Geostationary Coastal and Air Pollution Events

GPS - Global Positioning Satellite

GRACE - Gravity Recovery and Climate Experiment

GRIPS - Gamma-Ray Imager/Polarimeter for Solar

HEDS - Human Exploration Destination Systems

HERO — High-Energy Replicated Optics

HiRISE - High Resolution Imaging Science Experiment

HMaG - Heliospheric Magnetics

HyspIRI - Hyperspectral Infrared Imager

$\mathrm{Hz}$ - Hertz

IHM — Integrated Health Management

InGaAs - Indium Gallium Arsenide

INMS - Ion and Neutral Mass Spectrometer

INCA - Ion-Neutral Coupling in the Atmosphere

IXO - International X-ray Observatory

JAXA - Japanese Aerospace and Exploration Agency

LCAS - Low-Cost Access to Space

LIBS - Laser-Induced Breakdown Spectroscopy

LIMA — Long-range laser Induced Mass Analysis

LISA - Laser Interferometer Space Antenna

LIST — Lidar Surface Topography

LROC - Lunar Reconnaissance Orbiter Camera

MAHLI - Mars Hand Lens Imager

MCP - Microchannel Plate

Mdeg - Millidegree

MECA - Microscopy, Electrochemistry, and Conductivity Analyzer

MER - Mars Exploration Rovers

MKIDS - Microwave Kinetic Inductance Detectors

MSL - Mars Science Lab 


\section{Acronyms}

MSR — Mars Sample Return

NDE - Non-Destructive Evaluation

NEO - Near Earth Object

NEP — Noise Equivalent Power

NF - New Frontiers

NIR - Near Infrared

NRC - National Research Council

NuSTAR - Nuclear Spectroscopic Telescope Array

NW - New Worlds

O-Optical

ONSET - Origins of Near Earth Plasma

OR\&PE - Object Recognition and Pose Estimation

PATH - Precipitation and All Weather Temperature and Humidity

PNT — Position, Navigation, and Timing

PRF - Pulse Repetition Frequency

PSF - Point Spread Function

PVP - Photovoltaic Power

QE - Quantum Efficiency

RAT - Rock Abrasion Tool

RFI - Radio Frequency Interference

ROIC - Readout Integrated Circuit

SAIL — Synthetic Aperture Imaging Lidar

SAR - Synthetic Aperture Radar

$\mathrm{SA} / \mathrm{SPaH}$ - Sample Acquisition / Sample Processing and Handling

SCLP - Snow and Cold Land Processes

SEM - Scanning Electron Microscope

SEM - Space Experiment Module

SEPAT - Solar Energetic Particle Acceleration and Transport
SEU/SEL - Single Event Upset/Single Event Latchup

SIOSS - Science Instruments, Observatories, and Sensor Systems

SMD - Science Mission Directorate

SPICA - Science Investigation Concept Studies

SSE - Solar System Exploration

STP - Solar Thermal Propulsion

SWOT - Surface Water and Ocean Topography

TABS - Technology Area Breakdown Structure

TEPC - Tissue Equivalent Proportional Radiation Counter

TES - Transition Edge Sensors

THEMIS - Time History of Events and Macroscale Interactions during

Substorms

$\mathrm{THz}$ - TeraHertz

TID — Total lonizing Dose

TIR - Thermal Infrared

TPF-C - Terrestrial Planet Finder-Coronagraph

TPS - Thermal Protection System

T/R - Transmitter/Receiver

UAV - Unmanned Aerial Vehicle

UV - Ultraviolet

UVOIR - UV-Optical-near IR Telescope

VIS - Visible

WCL - Wet Chemistry Laboratory

WFE - Wall Plug Efficiency

WFOV - Wide Field of View

WFIRST - Wide-Field Infrared Survey Telescope

WFSC - Wavefront Sensing and Control

WINCS - Wind Ion-drift Neutral-ion Composition

WPT - Wireless Power Transmission

$\mathrm{XMM}$ - X-ray Multi-Mirror Mission

XRD - X-Ray Diffraction

$\mathrm{XRF}$ - X-ray Fluorescence 